\title{
8
}

\section{IVUS Guided PCI}

\author{
T. Kovarnik and J. Horak \\ 2nd Department of Medicine - Department of Cardiovascular Medicine, \\ First Faculty of Medicine, Charles University in Prague and \\ General University Hospital in Prague, \\ Czech Republic
}

\section{Introduction}

Intravascular ultrasound is a clinical tool that has been used in a complimentary manner to contrast angiography in order to enhance the procedure success rate and patient outcomes. Particularly, there are some specific situations where IVUS can be very useful and information from IVUS overcome angiography limitations. These situations can be divided into three parts:

- pre-interventional

- during intervention

- post-interventional

Pre-interventional use of IVUS can help with assessment of hemodynamic significance of stenosis (in this place must be emphasized, that IVUS can estimate hemodynamic significance only non directly, but with acceptable correlation with fractional flow reserve and nuclear stress test), precise anatomic analysis (type of bifurcation, plaque burden in ostial part of side branch or left main) and can help with device selection: precise measurement of diameters in reference segments for better sizing of balloon or stent, type of lesion preparation such as dilatation with cutting balloon or atherectomy devices in bulky lesions, drug eluting stent for lesions with high probability of small in stent area achievement.

IVUS can be used during intervention for assessment of lesion preparation (proper position of wire; especially it's relation to stent struts, effect of predilatation, assessment of side branch ostium) for guiding in more complicated intervention (left main stenting, navigation of wires during PCI of chronic total occlusion, trifurcation PCI).

Post-interventional use of IVUS can answer questions about result adequacy (stent expansion, stent apposition), angiographic filling defects ("hazy lesions") after PCI (edge dissection, thrombus formation, inadequate stent expansion, prominent calcification) and diagnosis of complications (dissection, geographic mismatch, plaque protrusion inside the stent, inadequate stent expansion or apposition). IVUS can in most cases also reveal the reasons behind development of complications after PCI such is in stent thrombosis (mainly inadequate stent expansion or edge troubles such as dissection, or uncovered lesion) or unexpected early in stent restenosis (inadequate stent expansion).

IVUS is a mandatory tool in the cardiac catheterization laboratory today. Like all medical equipments it should be used by an experienced investigator for better understanding of PCI mechanisms, prevention and solution of complications. Nowadays it is possible to use many semi-automatic softwares for border detection, but all of them must be corrected 
manually to avoid serious mistakes and misunderstanding. The best way how to do that is just routine use of IVUS.

\subsection{Limitation of angiography}

Angiography is a gold standard for assessment of atherosclerotic impairment of coronary arteries and for guiding of coronary intervention. On the other hand it has many limitations. Widely accepted decision making point for diagnosis of flow-limiting lesion is lumen diameter less than $50 \%$ of the reference segment. However, it has been proven that there is no correlation between angiography assessment and functional measurement by fractional flow reserve (FFR) in intermediate lesions between $40-70 \%{ }^{1}$.

Based on autopsy studies we know, that atherosclerosis is more often diffuse than focal process affecting coronary arteries. These findings are in good correlation with intravascular ultrasound. The reference segment, which is angiographically normal often contains atherosclerotic plaques visible on IVUS. From this point of view we are comparing less affected segments with more affected ones during angiography and thus underestimate stenosis significance ${ }^{2,3}$. Other challenging issues are assessment of ostial lesions and bifurcations.

\section{Quantitative lesion assessment}

\subsection{Technique}

The most important parameter for quantitative lesion assessment is minimal lumen area (MLA), which has the best correlation with presence of ischemia (see below).Common misunderstanding comes from the assessment of relative severity of stenosis in percentage of "normal" reference diameter. Angiographic stenosis assessment is based on comparison of lumen diameter in reference segment and lumen diameter in the lesion. The most corresponding IVUS parameter is lumen area stenosis (LAS), which is calculated as minimal lumen area in reference segment - minimal lumen area in lesion / minimal lumen are in reference segment. More frequently used description of stenosis is plaque burden (PB), computed as external elastic membrane area - lumen area / external elastic membrane area4. We must interrogate each frame and look for the smallest lumen area, use routinely nitrates before IVUS probe insertion for avoiding spasms. In case of uncertainty in lumen measurement, especially in present of soft plaque it is recommended to flush guiding catheter by saline for clearing of picture. Using of automatic pullback devices is not necessary for quantitative lesion assessment, but allows us to perform longitudinal diameters or volumetric analysis. Manual pullback is better for analysis of a precise part of plaque, where we can stop IVUS probe to obtain more frames from the region of interest. For imaging of aorto-ostial lesions it is necessary to retract the guiding catheter back into the aorta and verify that the path of the IVUS probe is still coaxial with the ostium of the vessel ${ }^{5}$.

\subsection{Indication for coronary intervention}

The main indication for coronary intervention is lesion producing myocardial ischemia, which can be detect either non-invasively by stress myocardial perfusion imaging (SPECT) or invasively by measurement of pressure gradient across stenosis (fraction flow reserve, FFR). IVUS measurement correlates with both of them (table one). 


\begin{tabular}{|c|c|c|c|c|}
\hline Author & Description & Methods & $\begin{array}{c}\text { No of } \\
\text { patients }\end{array}$ & Results \\
\hline Briguori $^{6}$ & $\begin{array}{l}\text { angiographic stenosis } \\
40-70 \%\end{array}$ & $\begin{array}{l}\text { IVUS vs. } \\
\text { FFR }\end{array}$ & 43 & $\begin{array}{ll}\text { FFR }<0.75 \text { correlates with: } \\
-\quad \text { MLA } \leq 4 \mathrm{~mm}^{2} \\
-\quad \text { MLD } \leq 1.8 \mathrm{~mm} \\
-\quad \text { PB }>70 \% \\
-\quad \text { Lesion length }>10 \mathrm{~mm}\end{array}$ \\
\hline Abizaid 7 & $\begin{array}{l}\text { patients indicated for } \\
\text { PCI }\end{array}$ & $\begin{array}{l}\text { IVUS vs. } \\
\text { CFR }\end{array}$ & 73 & $\begin{array}{l}\text { CFR } \geq 2 \text { correlates with: } \\
\text { MLA } \geq 4 \mathrm{~mm}^{2} \\
\text { - } \quad \text { MLD } \geq 2 \mathrm{~mm}\end{array}$ \\
\hline Nishioka $^{8}$ & $\begin{array}{l}\text { consecutive IVUS } \\
\text { examinations }\end{array}$ & $\begin{array}{l}\text { IVUS vs. } \\
\text { myocardial } \\
\text { SPECT }\end{array}$ & 79 & $\begin{array}{l}\text { Positive scan correlates with: } \\
-\quad \text { MLA } \leq 4 \mathrm{~mm}^{2} \\
-\quad \text { PB }>73 \% \\
-\quad \text { LAS }>59 \%\end{array}$ \\
\hline Takagi $^{9}$ & $\begin{array}{l}\text { consecutive IVUS } \\
\text { examinations }\end{array}$ & $\begin{array}{l}\text { IVUS vs. } \\
\text { FFR }\end{array}$ & 42 & $\begin{array}{l}\text { FFR }<0.75 \text { correlates with: } \\
-\quad \text { MLA } \leq 3 \mathrm{~mm}^{2} \\
-\quad \text { LAS }>60 \%\end{array}$ \\
\hline Lee $^{10}$ & $\begin{array}{l}\text { vessels smaller than } 3 \\
\mathrm{~mm}\end{array}$ & $\begin{array}{l}\text { IVUS vs. } \\
\text { FFR }\end{array}$ & 94 & $\begin{array}{ll}\text { FFR } & <0.75 \text { correlates with: } \\
- & \mathrm{MLA} \leq 2.0 \mathrm{~mm} 2 \\
- & \mathrm{PB} \geq 80 \% \\
\text { - } & \text { Lesion lenght } \geq 20 \mathrm{~mm}\end{array}$ \\
\hline Abizaid 11 & IVUS deferred PCI & IVUS & 300 & $\begin{array}{l}\text { Safe deferral of PCI correlates with: } \\
\text { - } \quad \text { MLA } \geq 4 \mathrm{~mm}^{2} \\
\quad \text { MLD } \geq 2 \mathrm{~mm}\end{array}$ \\
\hline Jasti $^{12}$ & $\begin{array}{l}\text { ambiguous left main } \\
\text { stenosis }\end{array}$ & $\begin{array}{l}\text { IVUS vs. } \\
\text { FFR }\end{array}$ & 55 & $\begin{array}{l}\text { FFR }<0.75 \text { correlates with: } \\
-\quad \text { MLA } \leq 5.9 \mathrm{~mm}^{2} \\
-\quad \text { MLD } \leq 2.8 \mathrm{~mm}\end{array}$ \\
\hline Abizaid $^{13}$ & $\begin{array}{l}\text { patients with } \\
\text { borderline left main } \\
\text { stenosis }\end{array}$ & IVUS & 122 & $\begin{array}{l}\text { MACE predictor : } \\
\quad \quad M L D \leq 3.0 \mathrm{~mm}\end{array}$ \\
\hline Kang ${ }^{14}$ & Consecutive patients & $\begin{array}{l}\text { IVUS vs. } \\
\text { FFR }\end{array}$ & 201 & $\begin{array}{l}\text { FFR }<0.80 \text { correlates with: } \\
\text { - } \quad \text { MLA } 2.4 \mathrm{~mm}^{2} \\
-\quad \mathrm{PB} \geq 79 \%\end{array}$ \\
\hline Ben Dor ${ }^{15}$ & $\begin{array}{l}\text { patients with } \\
\text { intermediate lesion } \\
\text { during QCA }\end{array}$ & $\begin{array}{l}\text { IVUS vs. } \\
\text { FFR }\end{array}$ & 84 & $\begin{array}{l}\text { FFR }<0.8 \text { correlates with: } \\
-\quad \text { MLA } 2.4 \mathrm{~mm}^{2} \mathrm{~mm} \text { for vessels } \\
\text { 2.5-3.0 } \mathrm{mm} \\
\text { - } \\
\text { - }\end{array}$ \\
\hline $\mathrm{Ahn}^{16}$ & $\begin{array}{l}\text { consecutive patients } \\
\text { with SPECT and } \\
\text { IVUS }\end{array}$ & $\begin{array}{l}\text { IVUS vs. } \\
\text { SPECT }\end{array}$ & 150 & $\begin{array}{l}\text { Positive scan correlates with: } \\
-\quad \text { MLA } 2.1 \mathrm{~mm}^{2}\end{array}$ \\
\hline PROSPECT $^{17}$ & $\begin{array}{l}\text { clinical follow-up } \\
\text { after ACS }\end{array}$ & $\begin{array}{l}\text { IVUS and } \\
\text { MACE }\end{array}$ & 700 & $\begin{array}{l}\text { Predictors for MACE: } \\
-\quad \mathrm{PB}>70 \% \\
-\quad \text { MLA }<4 \mathrm{~mm}^{2} \\
-\quad \text { TCFA }\end{array}$ \\
\hline
\end{tabular}

Abbreviations: CFR - coronary flow reserve, MACE - major cardiac adverse event, MLA - minimal lumen area, MLD - minimal lumen diameter, PB - plaque burden, PCI- percutaneous coronary interventions, QCA - quantitative coronary angiography, SPECT - single photon emission computed tomography, TCFA thin cap fibroatheroma

Table 1. Studies with IVUS evaluation of hemodynamic significance 
Based on older studies the following recommendations for IVUS detection of significant stenosis were established:

Main epicardial artery:

- $\quad$ MLA $<4 \mathrm{~mm}^{2}$

- $\quad$ MLD $<2 \mathrm{~mm}$

Left main:

- $\quad$ MLA $<6 \mathrm{~mm}^{2}$

- $\quad$ MLD $3 \mathrm{~mm}$

However, recently published studies do not support these cut-off values. Instead of clear cut-off points there are number of different recommended values, which do not seem to be useful for routine practice. Based on these findings we must conclude that IVUS is not suitable for assessment of haemodynamic significance of intermediate lesions. IVUS can be only used for exclusion of haemodynamicly significant lesion with MLA more than $4 \mathrm{~mm}^{2}$ for main epicardial arteries and more than $6 \mathrm{~mm}^{2}$ for left main. The haemodynamic significance of each lesion is caused not only by lumen area, but also by amount of viable myocardium supplied by this vessel and by presence or absence of collaterales. These facts simply cannot be examined solely by a morphologic modality like IVUS. On the other hand the PROSPECT trial ${ }^{17}$ with clinical endpoints confirmed, that large plaque compromising lumen to $4 \mathrm{~mm}^{2}$ and less, especially together with higher content of necrotic tissue is a risk factor for future events. These issues will be matter of further studies.

There are no data about IVUS criteria for hemodynamic significance in saphenous vein graft, but it is recommend to use a cut-off point MLA $4 \mathrm{~mm}^{2}$ for graft supplying one coronary artery and MLA $6 \mathrm{~mm}^{2}$ for graft supplying two arteries.

The precise assessment of hemodynamic significance is a crucial point, because the rate of ischemic events is $5-10 \%$ / year in significant lesion and less than $1 \%$ / year in non-ischemic lesion. Performing PCI in non-ischemic lesion increase risk of event to $2-3 \%$ / year ${ }^{18}$ and furthermore increases risk of periprocedural ischemia or myocardial necrosis during PCI.

Very old IVUS-skeptic sentence "If you want to stent, do IVUS" was quite recently supported by the work of Nam et al. ${ }^{19}$. Authors randomized 167 patients with intermediate coronary lesion between FFR guided (cut-off for PCI was FFR <0.8) and IVUS guided (cutoff MLA $<4.0 \mathrm{~mm}^{2}$ ) coronary intervention. PCI was performed in 33.7\% lesion in FFR arm and in $91.5 \%$ in IVUS arm. This difference was highly statisticaly significant $(\mathrm{p}<0.001)$. On the other hand we ourselves have a different experience with IVUS assessment in borderline lesions. We estimate that we are performing PCI in $40-50 \%$ of borderline lesions based on IVUS criteria, which is closer to FRR guided arm in this study. The finding of $91.5 \%$ frequency of MLA $<4 \mathrm{~mm}^{2}$ in lesion between $40-70 \%$ is in our eyes unrealistic.

\subsection{Assessment of left main (LM)}

Left main stenosis is a very important predictor for future cardiac events ${ }^{20}$. Angiographic assessment of LM is often complicated for overlapping branches and short or no reference segment and can lead to inappropriate estimates of lesion severity ${ }^{21}$. IVUS is more sensitive for left main atherosclerosis than angiography ${ }^{22}$. Suter et al. $^{23}$ found that in half of the patients with an inconclusive angiogram IVUS detects a significant stenosis. There is no difference for left main assessment during pullback from left anterior descending artery (LAD) or left circumflex artery (LCX) ${ }^{24}$. On the other hand for accurate assessment of ostial part of LAD and LCX is necessary to perform two pullbacks from both daughter vessels, 
because oblique view can overestimates lumen area ${ }^{25}$. The main target in left main bifurcation (like in other bifurcation) is to make a decision between one or two stent strategy. Pullback from just one daughter artery can answer the question whether the ostium of second branch is affected or not. For a more precise evaluation of minimal lumen area it is necessary to perform pullback from both daughter branches.

The precise coaxial position of guiding catheter is important for measurement or ostial left main stenosis, other than coaxial position of guiding catheter overestimates the lumen area. This type of inappropriate measurement can be detected by elliptical instead of spherical shape of ostial left main.

The ostium of LM can be influenced not only by atherosclerosis but also by external compression between enlarged pulmonary artery and aorta. This compression occur during systolic phase and lumen is enlarged during diastolic phase (figure 1,2). This finding can be seen in patients with severe pulmonary artery hypertension

According to our experience we recommend to perform all PCI's of LM with IVUS guidance. This is supported by results of MAIN-COMPARE registry, where IVUS guidance of left main PCI was superior to angiographic guidance ${ }^{26}$.

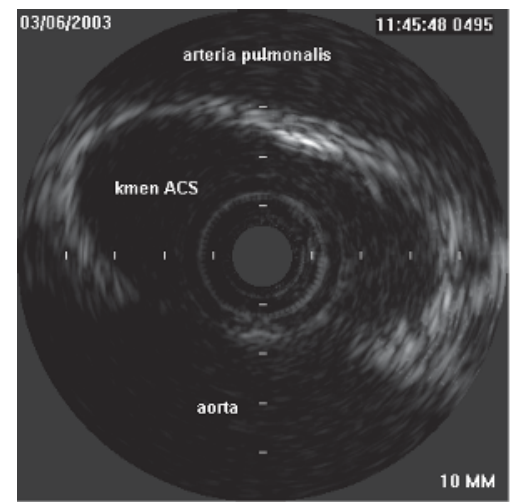

Fig. 1. External compression of left main during systolic phase of cardiac cycle. "Kmen ACS" means left main coronary artery.

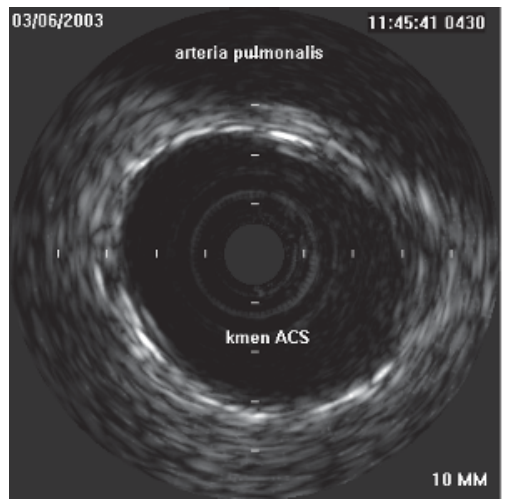

Fig. 2. No evidence of left main compression during diastolic phase of cardiac cycle. 


\section{Lesion morphology}

\subsection{IVUS in lesion with angiographic filling defect "hazy lesions"}

The main finding in so called hazy lesions is a defect in the contrast filling of coronary artery. The reasons for this include:

- $\quad$ eccentric calcification (figure 3)

- $\quad$ significant stenosis

- $\quad$ dissection (figure 4)

- $\quad$ thrombosis (figure 5)

- $\quad$ plaque rupture (figure 6)

- "flow phenomenon" (inadequate filling of big arteries during dye injection).

Thrombus is the most dangerous cause and many hazy lesions are treated like thrombuscontaining lesions with administration of IIbIIIa glycoprotein inhibitors, use of embolic protection devices or covered stents. However, real presence of intracoronary thrombosis is $50-60 \%$ of all hazy lesions ${ }^{27}$. Sensitivity of IVUS for thrombus is low (about $50 \%$ ), so from IVUS picture we simply cannot rule out the presence of thrombus (the highest sensitivity for thrombus has optical coherence tomography), but IVUS can confirm other causes and mainly causes which are not indicated fore PCI. This strategy decreases frequency of PCI in hazy lesions to $15-20 \% 28-30$

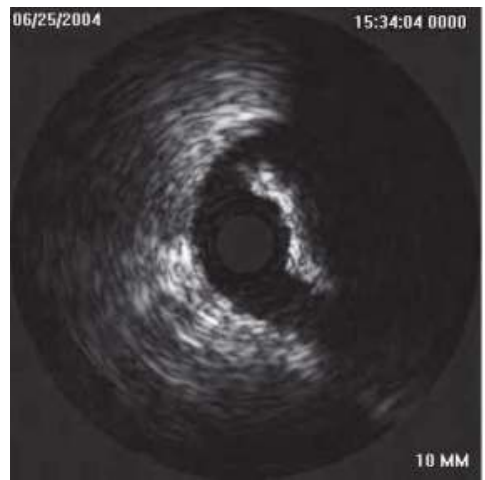

Fig. 3. Eccentric calcification.

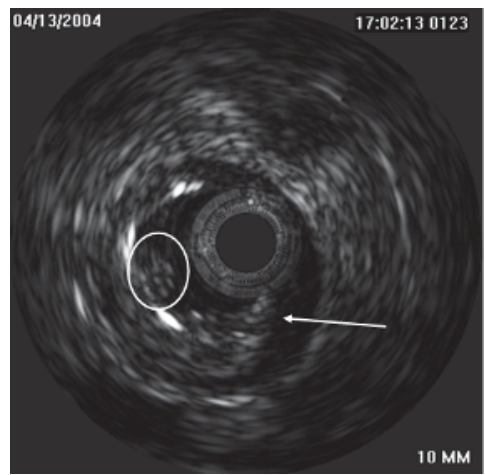

Fig. 4. Dissection with visible tear (arrow) and small thrombus (in circle). 


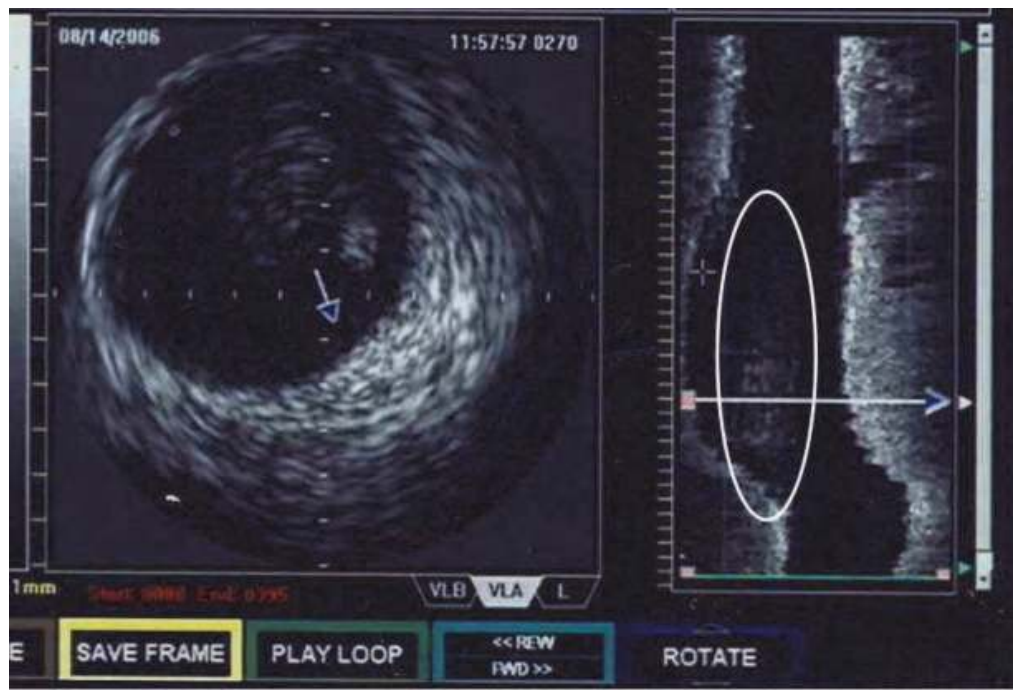

Fig. 5. Huge thrombus located on very small plaque causing acute coronary syndrome. Left side is cross sectional view, right side is longitudinal view (thrombus is located in the circle).

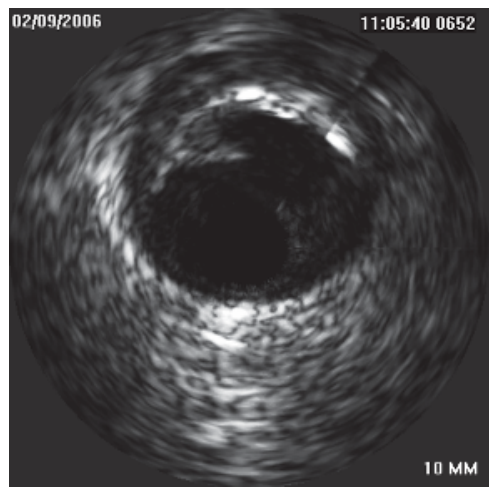

Fig. 6. Plague rupture with visible cavity after embolization of plaque mass. Patient with acute myocardial infarction.

\subsection{Aneurysms}

True aneurysm is defined as both an external elastic membrane (EEM) and lumen area 50\% larger than the proximal reference segment with intact three layers of vessel wall (figure 7). Lesions which seem to be the aneurysms from angiographic assessment are an true aneurysm in $37 \%$ of such findings. The rest are normal segments adjacent to plaque (53\%), complex atherosclerotic plaque $(16 \%)$ and pseudoaneurysm $(4 \%)^{31}$. Coronary pseudoaneurysm is due to rupture of vessel wall and in IVUS picture the three layers of vessel are not present, the shape of pseudoaneurysm is often irregular. From a practical point of view, pseudoaneurysms are seen only after coronary interventions causing trauma of vessel wall. 


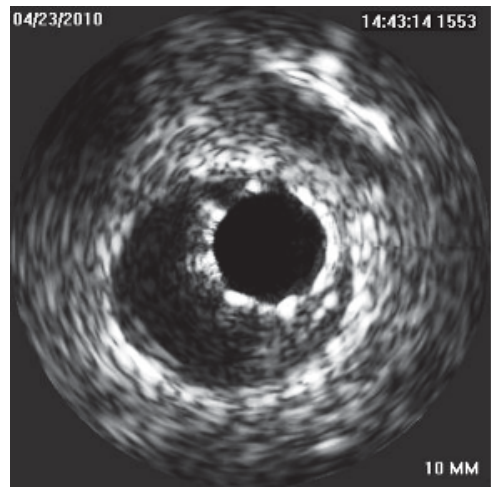

Fig. 7. Stent located in coronary aneurysm.

\subsection{True versus false lumen}

False lumen is created by dissection, either spontaneous or iatrogenic during coronary intervention (wire insertion or after balloon/stent dilatation). Dissections are treated by stent implantation and a flow non limiting dissection can be let to spontaneous healing. Stent insertion to the true lumen is a crucial point for proper treatment of dissection. True lumen can be identified by three-layered appearance and by origin of side branches. False lumen contains more echogenic blood flow ${ }^{5}$ (figure 8).

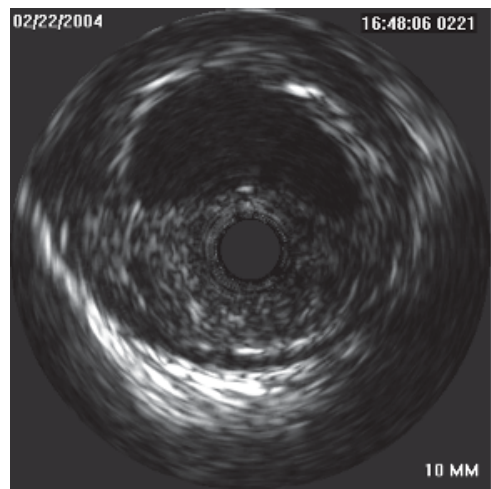

Fig. 8. Dissection with IVUS probe located in the false lumen with staying blood.

\section{Balloon angioplasty}

\subsection{The mechanism of balloon PCI}

The main mechanism of lumen enlargement during balloon dilatation is a plaque rupture, which enables lumen dilatation ${ }^{32}$. This finding was also confirmed by IVUS during in vivo studies, mainly in eccentric plaques (which are mainly present in coronary arteries). Another mechanism of lumen enlargement during balloon angioplasty is plaque compression and plaque redistribution, which are the main mechanism in concentric plaques ${ }^{33}$. 


\subsection{IVUS guided plain balloon PCI}

Several studies have been conducted in this field. Their results are summarized in table 2.

\begin{tabular}{|c|c|c|c|c|}
\hline study & target & restenosis & MACE & comments \\
\hline $\begin{array}{l}\text { CLOUT }^{34} \\
102 \text { pts. }\end{array}$ & $\begin{array}{l}\text { using larger balloon } \\
\text { than angiographicly } \\
\text { measured vessel } \\
\text { diameter }\end{array}$ & not declared & $1.9 \% *$ & $\begin{array}{l}\text { plaques compensated by } \\
\text { positive remodeling allow } \\
\text { use of aggressive dilatation } \\
\text { without risk of significant } \\
\text { dissections }\end{array}$ \\
\hline $\begin{array}{l}\text { Haase }^{35} \\
144 \text { pts. }\end{array}$ & $\begin{array}{l}\text { IVUS guided } \\
\text { balloon dilatation }\end{array}$ & $21 \%^{+}$ & $12^{+} \%$ & $\begin{array}{l}\text { use of larger balloon than } \\
\text { angiographic lumen } \\
\text { diameter is safe and with } \\
\text { low risk for restenosis }\end{array}$ \\
\hline \begin{tabular}{|l|} 
Schroeder \\
26 \\
252 pts.
\end{tabular} & $\begin{array}{l}\text { IVUS guided } \\
\text { balloon dilatation }\end{array}$ & $19 \%^{+}$ & $14^{+} \%$ & $\begin{array}{l}\text { small dissection are not } \\
\text { flow limiting } \\
\text { IVUS decreases number of } \\
\text { implanted stents } \\
\text { low restenosis comparable } \\
\text { with BMS }\end{array}$ \\
\hline $\begin{array}{l}\text { Abizaid } 37 \\
284 \text { pts. }\end{array}$ & $\begin{array}{l}\text { IVUS guided } \\
\text { balloon dilatation } \\
\text { and stent only in } \\
\text { case of } \\
\text { unsatisfactory result }\end{array}$ & $\begin{array}{l}8 \%^{+}-\mathrm{PCI} \\
16 \% \text { - stent }\end{array}$ & $\begin{array}{l}8 \%^{+}-\mathrm{PCI} \\
11 \% \text { - stent }\end{array}$ & $\begin{array}{l}\text { "stent like" effect after } \\
\text { IVUS guided balloon } \\
\text { dilatation has similar rate } \\
\text { restenosis like a BMS }\end{array}$ \\
\hline $\begin{array}{l}\text { BEST }^{38} \\
254 \text { pts. }\end{array}$ & $\begin{array}{l}\text { IVUS guided } \\
\text { aggressive PCI } \\
\text { (stent only when } \\
\text { necessary) vs. } \\
\text { routine stenting }\end{array}$ & \begin{tabular}{|l|}
$16.8 \% *$ \\
aggressive PCI \\
$18.1 \%$ stent
\end{tabular} & $\begin{array}{l}16 \% *- \\
\text { aggressive } \\
\text { PCI } \\
20 \% \text {-stent }\end{array}$ & $\begin{array}{l}\text { IVUS guided aggressive } \\
\text { PCI with provisional } \\
\text { stenting is safe and with } \\
\text { the same results like } \\
\text { routine stenting }\end{array}$ \\
\hline $\begin{array}{l}\text { SIPS } 39 \\
269 \text { pts. }\end{array}$ & $\begin{array}{l}\text { IVUS vs. angio } \\
\text { guided PCI }\end{array}$ & $\begin{array}{l}29 \% * \text { IVUS, } \\
35 \% \text { angio }\end{array}$ & $\begin{array}{l}30 \%{ }^{\nabla} \text { IVUS } \\
37 \% \text { angio }\end{array}$ & $\begin{array}{l}\text { same restenosis rate. } \\
\text { Lower TLR in IVUS group }\end{array}$ \\
\hline $\begin{array}{l}\text { Gaster }{ }^{40} \\
108 \text { pts. }\end{array}$ & \begin{tabular}{|l|} 
IVUS guided vs. \\
angio guided (IVUS \\
controlled) PCI
\end{tabular} & not declared & $\begin{array}{l}22 \% \cdot{ }^{\bullet} \text { IVUS } \\
41 \% \text { angio }\end{array}$ & $\begin{array}{l}\text { lower MACE in IVUS } \\
\text { guided group, better C/E3 } \\
\text { ratio in IVUS group }\end{array}$ \\
\hline $\begin{array}{l}\text { Meuller } 41 \\
\text { C/E } \\
\text { analysis of } \\
\text { SIPS study }\end{array}$ & $\begin{array}{l}\text { IVUS guided vs. } \\
\text { angio guided PCI }\end{array}$ & not declared & $\begin{array}{l}19.8 \%^{\nabla} \text { IVUS } \\
31.1 \% \text { angio }\end{array}$ & $\begin{array}{l}\text { lower MACE in IVUS } \\
\text { guided group, better C/E4 } \\
\text { ratio in v IVUS group }\end{array}$ \\
\hline $\begin{array}{l}\text { Colombo }{ }^{42 *} \\
130 \text { pts. }\end{array}$ & $\begin{array}{l}\text { IVUS guided } \\
\text { intervention in } \\
\text { lesions longer than } \\
15 \mathrm{~mm}\end{array}$ & $\begin{array}{l}25 \% * \text { IVUS } \\
39 \% \text { angio } \\
(p<0.05)\end{array}$ & $\begin{array}{l}22 \% * \text { IVUS } \\
38 \% \text { angio } \\
(\mathrm{p}<0.05)\end{array}$ & $\begin{array}{l}\text { Lower restenosis, MACE } \\
\text { and number of stents in } \\
\text { IVUS group }\end{array}$ \\
\hline
\end{tabular}

${ }^{+} 12$ months MACE, * 6 months $\mathrm{MACE}, \cdot 2,5$ year, $\mathrm{C} / \mathrm{E}$ cost/effectiveness ratio,${ }^{\nabla} 2$ years, TLR: target lesion revascularization, BMS: bare metal stents

* non randomized study, control group is composed by similar lesions

Table 2. IVUS guided coronary intervention 
The table 3 summarizes the strategy for choosing balloon diameter, acute complications and frequency of stenting in aforesaid studies.

\begin{tabular}{|c|c|c|c|c|}
\hline study & B/A ratio & equation for balloon diameter & $\begin{array}{l}\text { occurrence of } \\
\text { acute severe } \\
\text { dissection }\end{array}$ & stenting \\
\hline CLOUT $^{34}$ & $\begin{array}{l}1.3^{*} \\
0.8-1^{* *}\end{array}$ & mean MLDref + mean MVDref / 2 & $5 \%$ & not declared \\
\hline Haase $^{35}$ & $0.8-1$ ** & EEM in lesion & $11 \%$ & $0 \%$ \\
\hline Schroeder ${ }^{36}$ & $\begin{array}{l}1.4^{*} \\
0.88^{* *}\end{array}$ & $\begin{array}{l}\left(2 x E E M ~ r e f_{\text {prox }}+2 x \text { EEMref }_{\text {dist }}+\right. \\
2 x E E M \text { lesion }) / 6\end{array}$ & $12 \%$ & $2 \%$ \\
\hline Abizaid 37 & 1.34 * & $\left(\right.$ EEMmax $_{\text {lesion }}+$ EEM min $\left._{\text {lesion }}\right) / 2$ & $28 \%$ & $53 \%$ \\
\hline $\mathrm{BEST}^{38}$ & 1.25 * & $\left(\right.$ EEM ref $_{\text {prox }}+$ EEMref $\left._{\text {dist }}\right) / 2$ & $18 \%$ & $\begin{array}{l}44 \% \text { (in IVUS } \\
\text { group) }\end{array}$ \\
\hline SIPS39 & \begin{tabular}{|l|}
1.23 \\
IVUS* \\
1.03 angio
\end{tabular} & $\begin{array}{l}\text { MLDref + MVDref / } 2 \text { (in smallest } \\
\text { ref. segment) }\end{array}$ & $\begin{array}{l}3 \% \text { IVUS, } \\
3.2 \% \text { angio }\end{array}$ & $\begin{array}{l}49.5 \% \text { angio, } \\
49.7 \% \text { IVUS }\end{array}$ \\
\hline Gaster $^{40}$ & $\begin{array}{l}\text { not } \\
\text { declared }\end{array}$ & $\left(\right.$ MLAref $_{\text {prox }}+$ MLAref $\left._{\text {dist }}\right) / 2$ & not declared & $\begin{array}{l}85 \% \text { angio, } \\
87 \% \text { IVUS }\end{array}$ \\
\hline Meuller41 & $\begin{array}{l}\text { not } \\
\text { declared }\end{array}$ & not declared & not declared & $\begin{array}{l}49.5 \% \text { angio, } \\
49.7 \% \text { IVUS }\end{array}$ \\
\hline Colombo $42^{\circ}$ & 1.23 ** & EEM in lesion & $4.6 \%$ & $51.5 \%$ \\
\hline
\end{tabular}

Abbreviations: ref - reference segment, ref $_{\text {prox }}$ - reference proximal segment, ref $f_{\text {dist }}$ - reference distal

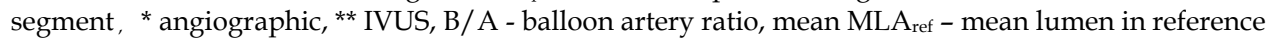

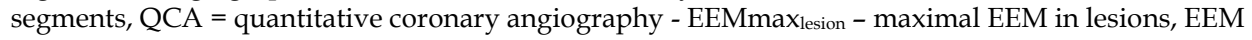

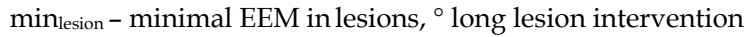

Table 3. Different strategies for choosing of balloon diameter.

The most frequent formula for choosing of balloon diameter is $\left(\right.$ EEMref $_{\text {prox }}+$ EEMref $\left._{\text {dist }}\right) / 2$

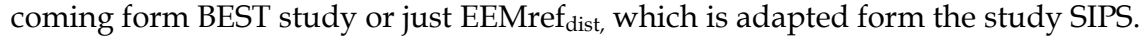

IVUS criteria for optimal result after balloon dilatation are listed in table 4 .

\begin{tabular}{|c|c|}
\hline study & criteria \\
\hline CLOUT $^{34}$ & MLA $\geq 65 \%$ mean MLA $_{\text {ref, }}$ no signs of flow limiting disection \\
\hline Haase $^{35}$ & increasing of MLA by at least $20 \%$ of EEM, no signs of flow limiting disection \\
\hline Abizaid 37 & MLA $\geq 65 \%$ mean MLA $_{\text {ref }}$, or $M L A \geq 6 \mathrm{~mm}^{2}$, no signs of flow limiting disection \\
\hline BEST38 $^{38}$ & $\begin{array}{l}\text { residual stenosis }<30 \% \text { (IVUS and angio), MLA }>6 \mathrm{~mm}^{2} \text {, no signs of flow } \\
\text { limiting disection }\end{array}$ \\
\hline SIPS39 & MLA $\geq 65 \%$ mean MLA $_{\text {ref, }}$ no signs of flow limiting disection \\
\hline Mueller 41 & $\begin{array}{l}\text { residual stenosis } \leq 35 \% \text {, MLA }>65 \% \text { meanMLA }_{\text {ref, }} \text { no signs of flow limiting } \\
\text { disection }\end{array}$ \\
\hline Colombo $42 *$ & MLA $\geq 50 \%$ EEM lesion, $\mathrm{MLA} \geq 5.5 \mathrm{~mm}^{2}$ \\
\hline
\end{tabular}

Abbreviations: mean $\mathrm{MLA}_{\text {ref }}$ - mean minimal lumen area in reference segment, QCA - quantitative coronary angiography, * long lesion intervention

Table 4. IVUS criteria of optimal result after balloon dilatation. 
Based on these studies with IVUS guided balloon dilatation we can summarize:

1. Using of larger balloons (balloon/artery ratio more than 1 according to angiographic assessment) is safe and without increased risk for acute severe complications. In hospital MACE were 1.4-3.9\%. Occurrence of significant dissection varies in large range, probably for different definition of this kind of complication. However, all authors declare low risk for sever acute dissection.

2. The rate of in stent restenosis is consistently low in all studies. It means that it is safe to avoid stent implantation after fulfilling of IVUS criteria for adequate results after balloon dilation. Surprisingly, higher in stent restenosis and higher MACE were found in some studies in patients with stent implantation compared to plain balloon dilatation. The reason for this finding is probably for bias; according to the study design stents were implanted to lesions with non satisfactory results after balloon dilatation or for treatment of complications after balloon dilatation. Higher occurrence of in stent restenosis and MACE is expected in these types of lesions.

3. The dark side of IVUS guided balloon angioplasty is a prolonged procedural time (5-13 minutes), increased X-ray time (2-3.6 minutes) and higher amount of contrast dye (12-34 $\mathrm{ml})^{38-41}$

4. Routine IVUS guided intervention can be beneficial from financial point of view. Optimal decision making decreases number of implanted stent and frequency of in stent restenosis and avoids peri-procedural complications from non-indicated coronary interventions ${ }^{40,41}$.

5. IVUS guided intervention should be used for avoiding stent implantation in patients unsuitable for dual antiplatelet therapy.

\section{Bare-metal stent implantation}

\subsection{Mechanism of lumen enlargement during stent implantation}

The most important mechanisms are plaque redistribution (inside stent) and plaque extrusion (outside stent) to the reference segments (more frequently to the distal one) ${ }^{43}$. Plaque redistribution and extrusion play role during restenosis in edge segment of stent ${ }^{44}$. Less important factors are plaque compression, plaque embolization and vessel enlargement (more in vessel with negative remodeling before PCI) ${ }^{43}$.

Final stent diameter is a result of interplay between pressure during implantation and vessel wall resistance. Declared stent diameters for different pressures during dilatation do not correlate with real stent diameter after implantation. These numbers for stent diameter come from in vitro tests in water and do not reflect real situation in the vessel. Costa et al.45 compared 200 drug eluting stent diameters from IVUS measurement immediately after implantation and declared stent diameter for nominal pressure. At least $90 \%$ of minimal lumen diameter for nominal pressure was reached only in $4 \%$ of stents. In stent area can be decreased immediately after implantation also by protrusion of plaque material through the struts $^{46}$ (figure9).

It is important to distinguish between stent expansion and stent apposition. Expansion means ratio between minimal stent area (MSA) and lumen area in reference segment. Inadequate expansion (figure 10) can be improved by high pressure postdilatation. Apposition reflects contact between stent struts and vessel wall. Inadequate apposition (figure 11) can be solved by low pressure dilatation with a bigger balloon. 


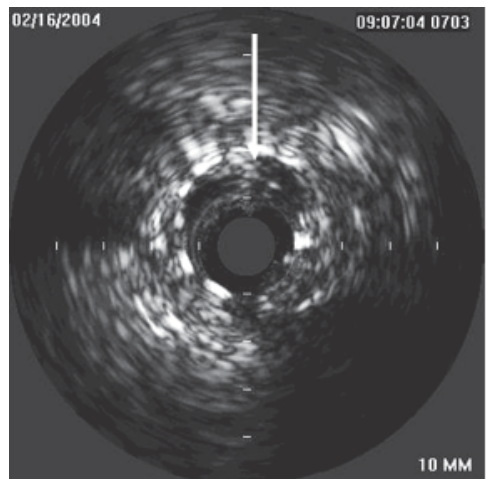

Fig. 9. Plaque protrusion through the struts to the lumen.

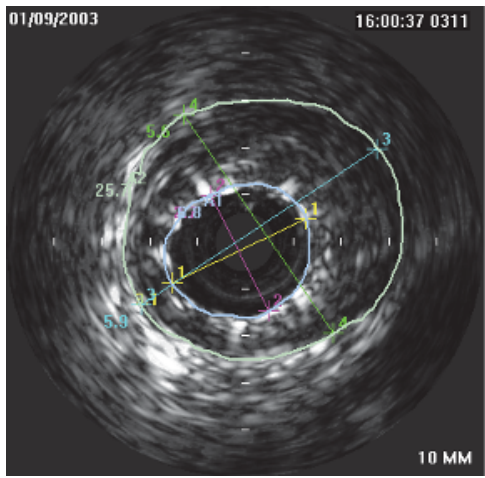

Fig. 10. Inadequate stent expansion.

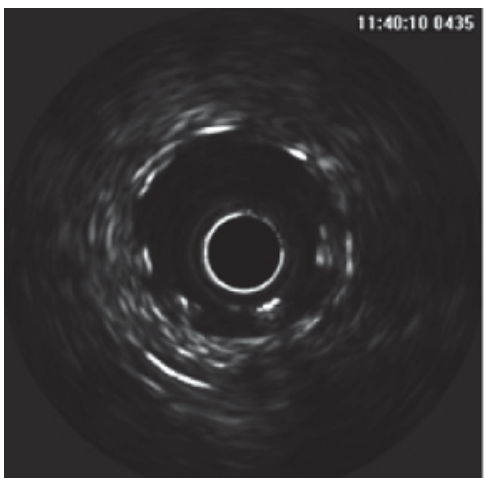

Fig. 11. Inadequate stent aposition

\subsection{High pressure stent dilatation}

The first implantations of Palmaz-Schatz and Gianturco-Roubin stents were complicated by high occurrence of subacute stent thrombosis. Colombo and coworkers started IVUS guided 
high pressure dilatation, which (together with dual antiagregation) decreased the rate of subacute stent thrombosis to $0.9 \% 47$. Routine use of high pressure dilatation (up to $20 \mathrm{~atm}$.) improves stent expansion and apposition without increasing acute complications ${ }^{48}$. Choi et al. ${ }^{49}$ found, that only $54 \%$ of angiographically adequatly expanded stents fulfill IVUS criteria for optimal stent expansion. Authors performed high pressure postdilatation and final MACE was only $11 \%$ in 6 months. This study confirms minimal stent area (MSA) as the most important risk factor of restenosis (figure 12).

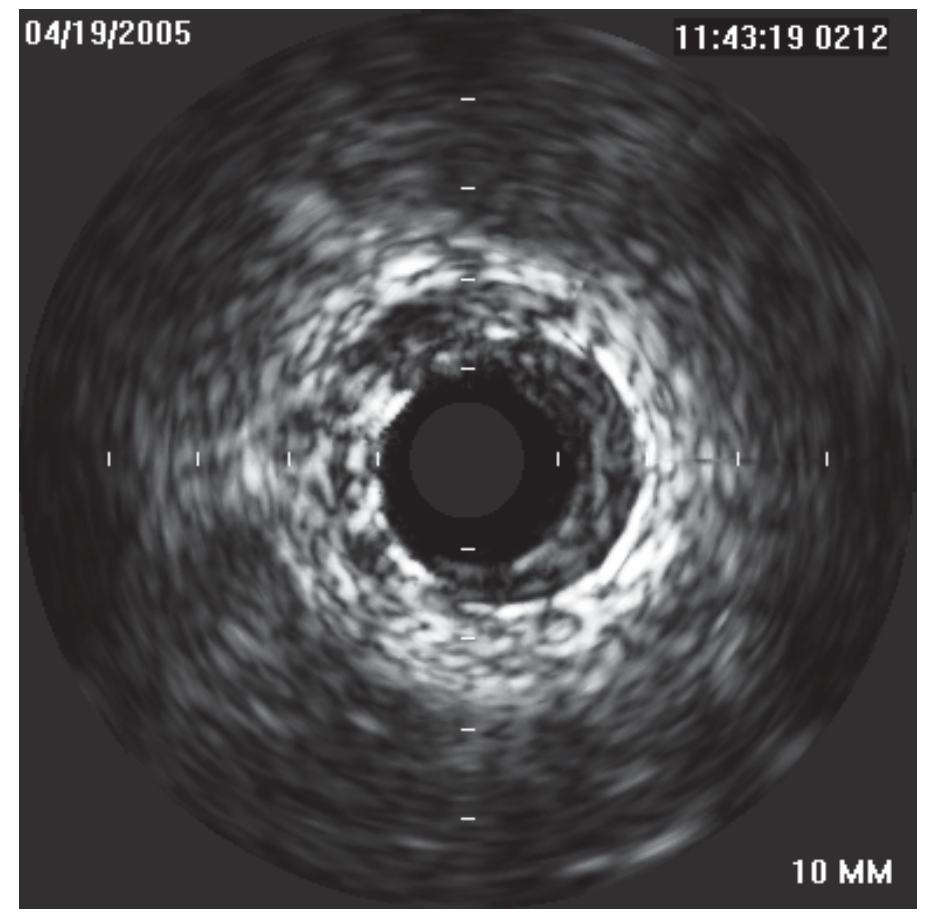

Fig. 12. In stent restenosis

\subsection{IVUS guided stent implantation}

There are many studies focusing on impact of IVUS guidance on stent implantation. They are summarized in table 5 .

Different criteria for optimal stent expansion were used in published trials, details are summarized in table 6.

\begin{tabular}{|l|l|l|l|l|}
\hline study name & study goal & restenosis & MACE & comments \\
\hline $\begin{array}{l}\text { MUSIC50 } \\
161 \text { pts. }\end{array}$ & $\begin{array}{l}\text { restenosis during } \\
\text { IVUS guided stent } \\
\text { implantation }\end{array}$ & $9.7 \%$ & $12.1 \%^{+}$ & $\begin{array}{l}\text { the second lowest } \\
\text { restenosis rate in studies } \\
\text { with BMS }\end{array}$ \\
\hline $\begin{array}{l}\text { Blasini51 } \\
105 \text { pts. }\end{array}$ & $\begin{array}{l}\text { IVUS vs. angio* } \\
\text { guided stent } \\
\text { implantation }\end{array}$ & $\begin{array}{l}20.9 \% \text { IVUS } \\
29.9 \% \text { angio } \\
(\mathrm{p}=0.033) * *\end{array}$ & not analyzed & $\begin{array}{l}\text { Restenosis 13.5\% in stent } \\
\text { fulfilling IVUS criteria }\end{array}$ \\
\hline
\end{tabular}




\begin{tabular}{|c|c|c|c|c|}
\hline study name & study goal & restenosis & MACE & comments \\
\hline $\begin{array}{l}\text { RESIST52 } \\
\text { study } \\
155 \text { pts. }\end{array}$ & $\begin{array}{l}\text { IVUS vs. angio } \\
\text { guided (IVUS } \\
\text { controlled) stent } \\
\text { implantation }\end{array}$ & $\begin{array}{l}22.5 \% \text { IVUS } \\
28.8 \% \text { angio } \\
(p=0.25)\end{array}$ & not declared & $\begin{array}{l}\text { IVUS guidance does not } \\
\text { decrease rate of in stent } \\
\text { restenosis }\end{array}$ \\
\hline $\begin{array}{l}\text { OPTICUS53 } \\
550 \text { pts. }\end{array}$ & $\begin{array}{l}\text { IVUS vs. angio } \\
\text { guided stent } \\
\text { implantation }\end{array}$ & $\begin{array}{l}24.5 \% \text { IVUS } \\
22.5 \% \text { angio } \\
(p=0.68)\end{array}$ & $\begin{array}{l}17.9 \% * \text { IVUS } \\
15.3 \% \text { angio } \\
(p=0.4)\end{array}$ & $\begin{array}{l}\text { IVUS guiding do not } \\
\text { decrease neither rate of in } \\
\text { stent restenosis nor } \\
\text { MACE }\end{array}$ \\
\hline $\begin{array}{l}\text { CRUISE }^{54} \\
499 \text { pts. }\end{array}$ & $\begin{array}{l}\text { IVUS vs. angio } \\
\text { guided (IVUS } \\
\text { controlled) stent } \\
\text { implantation }\end{array}$ & not declared & $\begin{array}{l}\text { TVR } \\
8.5 \% \text { in } \\
\text { IVUS group } \\
15.3 \% \text { in } \\
\text { angio group } \\
(p=0.019)\end{array}$ & $\begin{array}{l}\text { IVUS guided stenting } \\
\text { leads to lower TVR }\end{array}$ \\
\hline $\begin{array}{l}\text { Choi }{ }^{49} \\
278 \text { pts. }\end{array}$ & $\begin{array}{l}\text { IVUS vs. angio } \\
\text { guided stent } \\
\text { implantation }\end{array}$ & not declared & $\begin{array}{l}12 \%{ }^{+} \text {IVUS } \\
19 \% \text { angio } \\
(p=0,11)\end{array}$ & $\begin{array}{l}\text { IVUS leads to lower } \\
\text { periprocedural } \\
\text { complications with a } \\
\text { trend for lower TVR in } \\
\text { IVUS group }(\mathrm{p}=0.08)\end{array}$ \\
\hline $\begin{array}{l}\text { AVID } 55 \\
800 \text { pts. }\end{array}$ & $\begin{array}{l}\text { IVUS vs. angio } \\
\text { guided stent } \\
\text { implantation }\end{array}$ & not declared & $\begin{array}{l}18 \% \text { IVUS } \\
19 \% \text { angio }\end{array}$ & $\begin{array}{l}\text { IVUS leads to larger } \\
\text { lumen, but without any } \\
\text { effect on } 30 \text { days and } 12 \\
\text { months MACE }\end{array}$ \\
\hline $\begin{array}{l}\text { TULIP56 } \\
150 \text { pts. }\end{array}$ & $\begin{array}{l}\text { IVUS vs. angio } \\
\text { guided stenting of } \\
\text { long lesions }\end{array}$ & $\begin{array}{l}23 \%+{ }^{+} \text {IVUS } \\
46 \% \text { angio } \\
(p=0.008)\end{array}$ & $\begin{array}{l}6 \%^{+} \text {IVUS } \\
20 \% \text { angio } \\
(p=0.01)\end{array}$ & $\begin{array}{l}\text { Better clinical and } \\
\text { angiographic results in } \\
\text { IVUS group despite more } \\
\text { implanted stents }\end{array}$ \\
\hline $\begin{array}{l}\text { PRESTO57 } \\
796 \text { pts with } \\
\text { IVUS vs. } \\
8274 \text { with } \\
\text { angio }\end{array}$ & $\begin{array}{l}\text { IVUS vs. angio } \\
\text { guided stenting }\end{array}$ & not declared & $\begin{array}{l}\text { TVR } 13.8 \% \\
\text { IVUS } 12.2 \% \\
\text { angio }(\mathrm{p}=0.9)\end{array}$ & $\begin{array}{l}\text { Larger MLD in IVUS } \\
\text { group, but without } \\
\text { difference in MACE. } \\
\text { Criteria for adequate } \\
\text { IVUS stent expansion } \\
\text { was let only on operators } \\
\text { discretion }\end{array}$ \\
\hline $\begin{array}{l}\text { DIPOL } 58163 \\
\text { pts. }\end{array}$ & $\begin{array}{l}\text { IVUS vs. angio } \\
\text { guided stenting }\end{array}$ & $\begin{array}{l}10 \% \text { IVUS } 27 \% \\
\text { angio }\end{array}$ & $\begin{array}{l}7.3 \% \text { IVUS } \\
16 \% \text { angio }\end{array}$ & $\begin{array}{l}\text { Best results for IVUS } \\
\text { guided in randomized } \\
\text { trial }\end{array}$ \\
\hline $\begin{array}{l}\text { Gaster } 55,60 \\
108 \text { pts }\end{array}$ & $\begin{array}{l}\text { IVUS vs. angio } \\
\text { guided stenting }\end{array}$ & $\begin{array}{l}16 \% \text { IVUS } \\
25 \% \text { angio }\end{array}$ & $\begin{array}{l}22 \% \text { IVUS } \\
41 \% \text { angio }\end{array}$ & $\begin{array}{l}\text { IVUS guiding is cost } \\
\text { saving }\end{array}$ \\
\hline
\end{tabular}

* non randomized trial, angio group is a historic control, ${ }^{* *}$ IVUS criteria was fulfilled in $49.5 \%$ patients and in these ones was restenosis $13.5 \%,{ }^{+} 6$ months, ${ }^{*} 12$ months, ${ }^{\bullet} 9$ months, TVR - target vessel revascularization

Table 5. Trials assessing IVUS guided stent implantation. 


\begin{tabular}{|c|c|}
\hline study & criteria for optimal expanded stent \\
\hline Katritsis 61 & $\begin{array}{l}\text { full stent apposition, MSA } \geq 90 \% \text { meanMLA } \text { ref, }_{\text {, symmetrical expansion (min }} \\
\text { SD } / \text { maxSD }>0.7 \text { ) (correlation with FFR 0.94) }\end{array}$ \\
\hline Fearon $^{62}$ & MSA $7 \mathrm{~mm}^{2}$ (correlates with FFR 0.96) \\
\hline Hanekamp63 & $\begin{array}{l}\text { full stent apposition, } \mathrm{MSA} \geq 90 \% \text { meanMLA } \mathrm{A}_{\text {ref, }} \text { or MSA } \geq 100 \% \text { minMLA }_{\text {ref, }} \\
\text { symetrical expansion }(\operatorname{minSD} / \operatorname{maxSD}>0.7) \text {, (correlation with FFR 0.94) }\end{array}$ \\
\hline Gorge $^{48}$ & symmetrical stent expansion, $\mathrm{MSD} \geq 3 \mathrm{~mm}$, full apposition \\
\hline Choi 49 & $\begin{array}{l}\text { complete stent apposition, MSA } \geq 80 \% \text { distalMLA } \mathrm{A}_{\text {ref }} \text {, symmetrical expansion } \\
(\mathrm{minSD} / \operatorname{maxSD}>0.8)\end{array}$ \\
\hline Colombo 47 & full stent apposition, $\mathrm{MSA} \geq \operatorname{minMLA}_{\mathrm{ref}}, \mathrm{MLA}>60 \% \mathrm{MLA}_{\text {ref }}$ in edge regions \\
\hline Ahmed $^{44}$ & complete stent apposition, MSA $\geq 80 \%$ meanMLA $_{\text {ref }}$ or MSA $7.5 \mathrm{~mm}^{2}$ \\
\hline MUSIC50* & $\begin{array}{l}\text { - } \\
\text { complete stent apposition, } \mathrm{MSA} \geq 90 \text { meanMLA }_{\text {ref }} \text { or } \geq 100 \text { minMLA }_{\text {ref }} \\
\text { in case of }>9 \mathrm{~mm}^{2}: \\
\text { MSA in proximal part of stent } \geq 90 \% \text { proxMLA } \\
\text { symmetrical expansion (minSD } / \text { maxSD }>0.7 \text { ) }\end{array}$ \\
\hline Blasini 51 & $\begin{array}{l}\text { full stent apposition, MSA }>8 \mathrm{~mm}^{2} \text {, or MSA } \geq 90 \% \text { meanMLA }_{\text {ref, }} \text { complete } \\
\text { coverage of dissection }\end{array}$ \\
\hline RESIST52 & MSA $\geq 80 \%$ meanMLA ref \\
\hline OPTICUS53 & MUSIC criteria, residual stenosis les than 10\% (angiographically) \\
\hline CRUISE54 & residual stenosis < 10\% (angiographically) \\
\hline AVID 55 & full stent apposition, residual stenosis $<10 \%$, no signs of dissection \\
\hline TULIP56 & complete stent apposition, $\mathrm{MSD} \geq 80 \%$ meanMLD ref, $\mathrm{MSA} \geq 90 \%$ distalMLA $\mathrm{A}_{\text {ref }}$ \\
\hline
\end{tabular}

Abbreviations: SD - stent diameter, meanMLA $\mathrm{A}_{\text {ref }}$ - mean minimal lumen area in reference segments, minMLA $_{\text {ref }}$ minimal - minimal lumen area i reference segments, MSD - minimal stent diameter, MSA minimal stent area

* all criteria were fulfilled in $81 \%$ patients and these patients were treated only with acetylosalycilic acid and rate of subacute stent thrombosis was $1.3 \%$

Table 6. Different criteria for optimal stent expansion.

The main goal for IVUS guidance of stent implantation is a larger minimal stent diameter and minimal stent area. Further improvement is seen in strut apposition. Minimal stent area is the most important risk factor for development of in stent restenosis (ISR). The cut-off point for risk of ISR is $8 \mathrm{~mm} 2$ in vessel $\geq 3 \mathrm{~mm}$ and $6 \mathrm{~mm} 2$ in vessel $<3 \mathrm{~mm}^{64}$. The rate of in stent restenosis according to achievement of different IVUS parameters is summarized in table 7 .

\begin{tabular}{|c|c|}
\hline criteria & restenosis rate \\
\hline MSA $>9 \mathrm{~mm}^{2}$ & $11 \%$ \\
\hline MSA $>9 \mathrm{~mm}^{2}$ a MSA $\geq 80 \%$ meanMLA ${ }_{\text {ref }}$ & $12.5 \%$ \\
\hline MSA $\geq 55 \%$ meanEEM $_{\text {ref }}$ & $17 \%$ \\
\hline MSA $\geq 90 \%$ meanMLA ref & $21 \%$ \\
\hline MSA $\geq 90 \%$ distal MLA $_{\text {ref }}$ & $22 \%$ \\
\hline
\end{tabular}

Abbrevation: MSA - minimal stent area

Table 7. Risk of ISR and achievement of different IVUS parameters. 
The lowest rate of ISR is in stents with MSA $>9 \mathrm{~mm}^{2}$. However, for achievement of this MSA it is necessary to use a $3.5 \mathrm{~mm}$ stent (with ideal stent area $9.6 \mathrm{~mm}^{2}$ ), because $3 \mathrm{~mm}$ stent has an ideal stent area $7.1 \mathrm{~mm}^{2}$. MSA $8 \mathrm{~mm}^{2}$ as a sufficient post-stenting area was confirmed by Hoffmann et al ${ }^{65}$. They found a mean MSA in stent with ISR $7.1 \mathrm{~mm}^{2}$ and $8.1 \mathrm{~mm}^{2}$ in stents without development of ISR. This cut-off MSA is also a predictor for development of ISR in long stents ${ }^{66}$. Further predictors for ISR are:

- length of stents67

- $\quad$ strong calcification 68

- $\quad$ bulky plaque compensated by positive vessel remodelation69-72 (figure 13)

- $\quad$ plaque protrusion through the stent struts 73

MSA is the most important factor for development of in stent thrombosis (IST) $)^{74}$. The incidence of this feared complication is less than $0.5 \% 74$.

Parise et al..$^{75}$ published recently a meta-analysis of all randomized studies comparing IVUS guided vs. angio guided stenting in pre-drug-eluting stent era. They concluded that IVUS guided stenting significantly lowered the 6-month angiographic restenosis rates, 12-month revascularization and MACE rate. They did not find any effect of IVUS guidance on death or myocardial infarction.

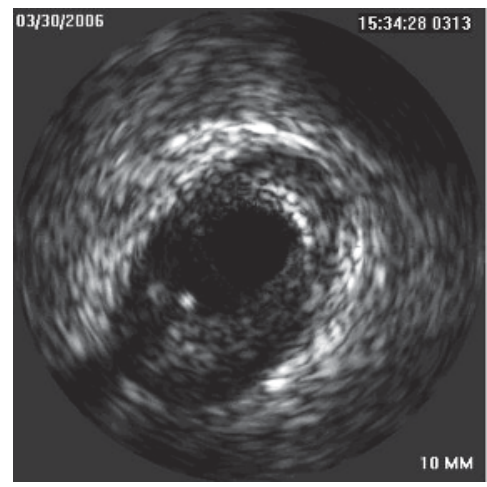

Fig. 13. Large plaque compensated by positive vessel remodeling

\section{Drug-eluting stent implantation}

Drug eluting stents (DES) significantly reduce the risk of in stent restenosis compared to BMS76-78. IVUS guidance seemed to be needless for DES excellent results. However, several issues are still a problem even with the use of DES and some of them, like in stent thrombosis, seem to be more important than in BMS .

In stent restenosis still exists and it's incidence is $5-10 \%$ in selected populations ${ }^{79-80}$. It seems to be more pronounced in paclitaxel eluting stent than sirolimus eluting stents ${ }^{81}$. Minimum stent area that best separates restenosis from no restenosis in DES is between $5.0-6.0 \mathrm{~mm}^{2} 82,83$.

The occurrence of in stent thrombosis (IST) in DES is $1-1.5 \% 84$. The main cause of IST is stent under expansion. DES with further IST development showed significantly lower MSA (4.3$4.6 \mathrm{~mm}^{2}$ ). Further risk factor for IST development is residual edge stenosis, defined as a MLA $<4 \mathrm{~mm}^{2}$ and a plaque burden $>70 \% 85,74$. These risk factors remain the same like in BMS, where the presence of dissection, thrombus or tissue prolaps into the stent were 
recognized as further risk factors for IST development ${ }^{86,87}$. The occurrence of IST in BMS is estimated at $0.9 \% 88$.

The risk factors for IST development can be revealed by IVUS control after stent implantation. Roy et al. ${ }^{89}$ published study, where IVUS guidance of DES implantation reduced development of IST in 30 days ( $0.5 \%$ in IVUS vs. $1.5 \%$ in angio guided group) and 12 month (0.7\% in IVUS vs. $2.0 \%$ in angio guided group), as well as decreased need for revascularization and MACE in 30 day. Unfortunately, there were no clear criteria for adequate stent deployment in this study. Claessen et al. 90 published a study comparing angio and IVUS guidance in 1504 sirolimus eluting stent implantation in the MATRIX (Comprehensive Assessment of Sirolimus-Eluting Stents in Complex Lesions) registry. They found significantly lower occurrence of myocardial infarction and combined endpoint of myocardial infarction and death in IVUS guided group. Hur et al. ${ }^{91}$ published their enormous registry of angio (3744 patients) versus IVUS (4627 patients) guided stent implantation. The main finding of this study is lower mortality in IVUS guided group. This result was seen mainly in DES implantation, because mortality in BMS group was the same. However, the explanation of this interesting finding is not clear, because the occurrence of myocardial infarction, target vessel revascularization and in stent thrombosis was without significance differences between angio and IVUS group. Based on aforesaid trials we can conclude, that IVUS guidance of DES implantation probably improves patient's outcome, but further trials are needed for better understanding of this phenomenon.

Important phenomenon in DES implantation is incomplete stent apposition (ISA), which means, that at least one strut is not adequately apposed to vessel wall. One should distinguish between acute stent malapposition, which is present immediately after stent implantation, late stent malapposition (LSM), which can be persistent, it means that acute malapposition is not healed and late-acquired stent malapposition (LASM), which develops despite normal finding after stent implantation. The mechanism of LASM development is not known, several mechanisms are thought:

- $\quad$ positive vessel remodeling leading to vessel enlargement

- $\quad$ decrease of plaque volume behind the stent caused by antiproliferative effect of DES

- $\quad$ thrombus dissolution after PCI of thrombus containing lesions

The frequency of LSM is not clear, a quite broad range for this phenomenon was published $(4-21 \%)^{92,93}$. LSM is not found only in DES, but also in BMS, where it's incidence is 4.4$5.4 \% 94,95$. Empty space behind stent struts can lead to decrease of blood flow in this region and cause development of thrombosis. However, this theoretic concept was not proved in any study following the natural course of patients with LSM. Hong et al. ${ }^{96,97}$ did not find any clinical adverse event in patient with LSM during 10 months follow up. On the other hand Cook et al. ${ }^{98}$ published correlation between LSM and very late IST. Moreover, Hassan et al. ${ }^{99}$ published meta-analysis of 17 randomized trials focusing on LSM in BMS as well as DES. They found four times higher risk of LASM in patients with DES compared to BMS and LSM (acquired or persistent) increased significantly risk for (very) late in stent thrombosis (OR 6,51).

A rare complication $(1.25 \%)$ of DES implantation is development of coronary aneurysm. The definition of aneurysm is focal enlargement of vessel lumen, which is $50 \%$ larger than adjacent reference vessel. Coronary aneurysm is not a benign finding as up to $40 \%$ of patients needed revascularization in a study done by Alfonso et al. ${ }^{100}$. 


\section{Ivus guided pci in specific situation}

\section{1 $\mathrm{PCl}$ of bifurcation lesions}

$\mathrm{PCI}$ in bifurcation is a more challenging procedure with a risk of compromising flow in side branch (SB) and with higher rate of restenosis. The "classical" mechanisms of worsening flow in side branch were thought 101

- plaque compression in ostial part of SB during dilatation in main vessel (MV). The presence of diffuse ostial plaque (figure 14) has higher risk for TIMI 2 flow after stenting than presence of eccentric plaque ${ }^{102}$

- plaque shifting from the MV to SB during dilatation in MV ("snow-plow" phenomenon)

- $\quad$ interposition of stent struts across the ostium of SB

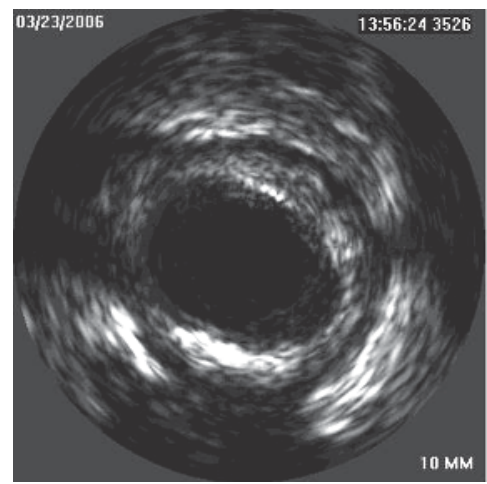

Fig. 14. Bifurcation with diffuse impairment of side branch ostium.

Plaque in bifurcation is mainly localized in counter carina ${ }^{103}$. However, Medina et al.104 proved the presence of plaque also in carina. They found plaque in $32 \%$ of bifurcations and moreover, in $16 \%$ of bifurcations the plaque in the carina was larger than in counter carina area. Furthermore, authors showed, that the mechanism of ostial SB damage after stent implantation in the MV was always due to displacement of the carina and no cases of plaque shifting were found. Further interesting finding of this study is that plaque in the carina was greater in the bifurcations that had maximum stenosis located distal to the carina in the MV. Authors explain this finding by hypothesis of different flow velocity patterns in these lesions. Atheroprotective high shear stress can be transformed into an atero-prone low shear stress. Authors describe less damage of SB ostium after stenting of lesions with plaque at the carina. Plaque probably makes carina more resistant and does not allow carina shifting, which is now thought to be a dominant mechanism causing damage to the SB ostium when a stent is implanted into the MV.

Important contribution for planning of PCI in bifurcations is a study done by Costa et al 105 . They found that the part of MV just behind the origin of SB (so called "lower diamond" or "polygon of confluence") is more prone for small minimal stent area after dilatation of SB ostium, which is not reverted even after kissing balloon dilatation. Kang et al. ${ }^{106}$ published a study assessing the IVUS predictors for side branch compromise after single-stent crossover technique and found two predictors for post stenting FFR $<0.8$ : MLA $2.4 \mathrm{~mm}^{2}$ and PB $>51 \%$ in SB ostium. 
Main role of IVUS guidance in bifurcation PCI is precise assessment of atherosclerotic burden of main vessel, carina, ostial part of side branch and the choice of the best strategy based on these findings. IVUS can improve the choice of ideal stent diameter, because bifurcation area is frequently affected by negative remodeling, which is not visible in routine angiography 107 .

IVUS should be used of most cases of uncertain atherosclerotic distribution in bifurcation lesions, because in case of favorable finding in SB ostium a planned complex procedure can be converted to a simple one with just stenting of MV.

\subsection{PCl of chronic total occlusion}

The main disadvantage for IVUS guidance of chronic total occlusion (CTO) interventions is the lateral view of IVUS probe. A prototype of forward-looking IVUS system was developed, but till now it has never been commercialized. IVUS can help during wire introduction to the proximal fibrous cap of CTO in presence of side branch just proximal to occluded segment. IVUS is introduced to this side branch and from this location the wire reaching the origin of total occlusion can be visualized (figure 15). IVUS also can help with re-introduction of wire from false lumen to the true lumen, but it is necessary to introduce the IVUS probe to the false lumen after its predilatation with small balloon ${ }^{108}$. This technique unfortunately increases risk of vessel perforation.

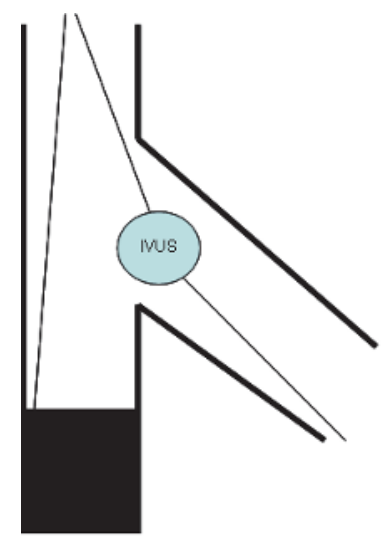

Fig. 15. Schema of IVUS guidance during PCI of chronic total occlusion.

\section{Complications}

\subsection{Dissections}

Dissections are tears in the plaque that are parallel to the vessel wall with blood flow in the false lumen and tend to occur at the junction of elements with different compliance (plaque and normal vessel wall, edges of stents) (figure 16, 17). Dissections can be described as proximal or distal to the lesion; epicardial or myocardial; and according to length, circumferential arc, depth, lumen compromise, bulkiness and mobility of the flap ${ }^{5}$. The major predictor of an unfavorable prognosis of dissection is a decreased blood flow in affected coronary artery. This situation must be solved immediately. IVUS can also detect dissections, which are not visible on angiography and it is questionable how many of them should be 
treated. Nishida et al. 109 followed 124 patients with non-flow limiting dissection (65\% of them were after stenting). They found that dissection can be let untreated if the residual lumen is more than $6 \mathrm{~mm}^{2}$ and lumen area is more than $40 \%$ of the EEM area. The edge dissections after stenting, which can be visualized only by IVUS and are not apparent during angiography, are not indicated for any intervention, because of good prognosis by spontaneous healing ${ }^{110}$.

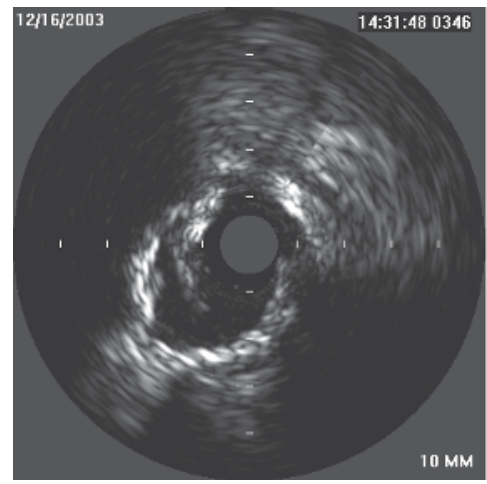

Fig. 16. Dissection with tear reaching to the media.

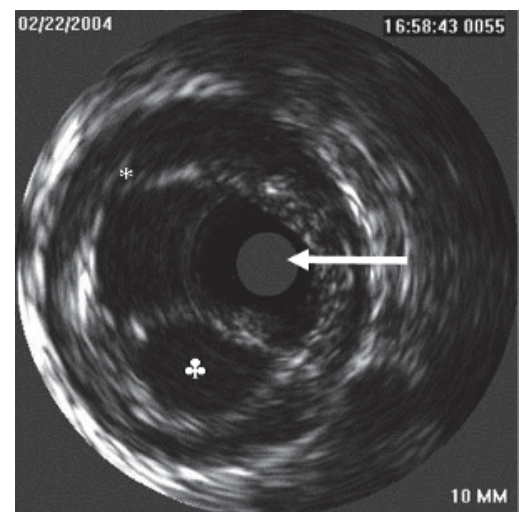

Fig. 17. Complex dissection with IVUS probe in true lumen, one false lumen is located down to true lumen (*) and another entry to the false lumen is located above the true lumen $(*)$.

\subsection{Intramural hematomas}

Intramural hematoma is a variant of a dissection. The angiographic appearance ranges from a dissection, thrombus, and abrupt closure to non-significant abnormality. The EEM expands outwards and the intima is pushed inwards and straightens to cause lumen compromise (figure 18). Blood accumulates in the space caused by the split in the media and becomes static and echogenic. The hematoma can propagate antegrade or retrograde, but tends to be stopped by branches or severely diseased parts of the vessel (particularly calcified plaques). In a study, which included more than 1000 patients with IVUS control after PCI, an intramural hematoma was found in $6.7 \%$ with a high rate of clinical events 5,111 . 


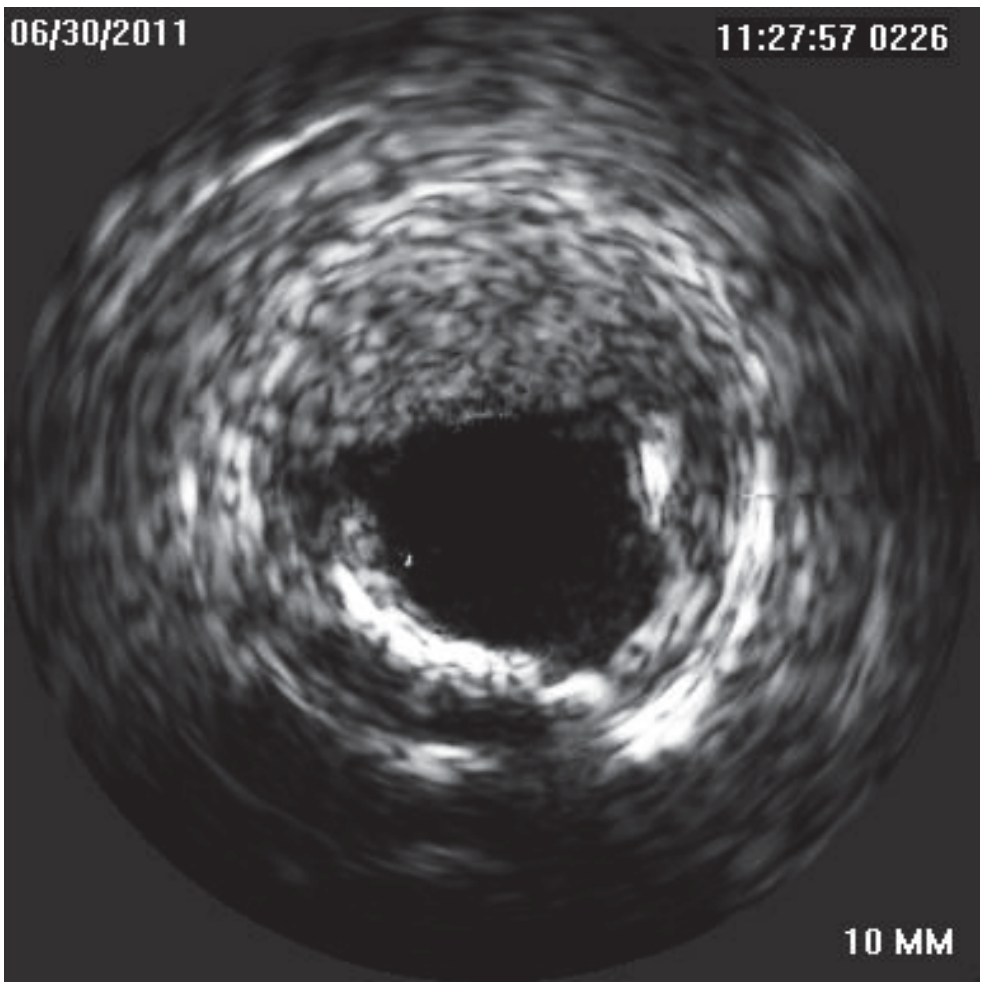

Fig. 18. Intramural haematoma. Bleeding to the media and adventicia is compromising lumen.

\subsection{Haziness}

IVUS is a very useful tool for diagnosis of underlying processes causing a hazy appearance of lesions after PCI. The most common findings are large residual plaque burden, dissection and plaque protrusion. Rarely stent deformation or intramural hematoma can be found. Intimal wrinkling can occur when a vessel is straightened by a guidewire. It reverses when the wire is removed or when the stiff wire is exchanged for a soft one. A narrowing of the lumen with a straightening of a normal-looking intima, behind which is an echolucent zone can be seen in IVUS picture. Discontinuity of the EEM can be found, which is caused by simultaneously visualized both sides of fold in the artery. Finally, it is important to recognize an angiographic pseudo complication with normal IVUS finding 5 .

\section{Conclusions}

Based on studies focusing on IVUS guidance of stent implantation we can summarize:

1. Based on IVUS criteria we can probably safely defer PCI

2. Satisfactory IVUS finding after balloon dilatation has same results as a stenting with BMS

3. IVUS guidance decreases angiographic restenosis and consequently the revascularization and MACE rate 
4. IVUS guidance decreases rate of in stent thrombosis

5. Contraindication for IVUS guidance are small vessels, tortuous vessels and degenerated vein grafts

This summary raises the question why IVUS is not used routinely during all PCI procedures. The answers can be divided into two groups. First there are the lessons we have learned from IVUS and this phenomenon is known as an "IVUS eye". We know that vessels are bigger than they look on angio and we are using bigger stents than before IVUS studies. We know, that without high pressure post dilatation we are not able to achieve sufficient stent diameter despite information derived from manufacturer's compliance charts. These factors dramatically contributed to lowering of in stent restenosis and therefore it is difficult to find statistical significant difference between IVUS and angio guided interventions, like it was described in OPTICUS trial. However, IVUS is still irreplaceable during investigation of unclear finding during angiography (like hazy lesions) or after stenting. IVUS is especially recommended during PCI of left main, the last remaining coronary artery and PCI in patients who are contraindicated for dual antiplatelet therapy for avoiding stent implantation. Furthermore, precise assessment of bifurcation can change the strategy from more complex to simple one in case of low risk profile of ostial part of side branch The group of arguments against routine use of IVUS during PCI is cost of IVUS catheter, longer procedural time and higher contrast dye consumption. Moreover IVUS guided PCI are more complex and need more skills than angio guided ones. However, in indicated cases IVUS guidance undoubtedly improves short as well as long term of coronary interventions.

\section{References}

[1] Pijls NH, de Bruyne B, Peels K et al, Measurement of Fractional Flow Reserve to Assess the Functional Severity of Coronary-Artery Stenoses. NEJM 1996; 334: 1703-1708

[2] Topol EJ, Nissen SE.Our preoccupation with coronary luminology. The dissociation between clinical and angiographic findings in ischemic heart disease. Circulation 1995;92:2333-2342

[3] Vlodaver Z, Frech R, Van Tassel RA, et al. Correlation of the antemortem coronary angiogram and the postmortem specimen. Circulation 1973;47:162-169

[4] Mintz GS, Nissen SE, Anderson WD, Erbel R, Fitzgerald PJ, Pinto FJ, Rosenfiels K, Siegel K, Tuzcu EM, Yock PG. American College of Cardiology Clinical Expert Consensus Document on Standards for Acquisition, Measurement and Reporting of Intravascular Ultrasound Studies (IVUS) A Report of the American College of Cardiology Task Force on Clinical Expert Consensus Documents. JACC 2001;37:1478-1492

[5] Mintz GS. Intracoronary ultrasound. Taylor \& Francis 2005

[6] Briguori C, Anzuini A, Airoldi F, et al. Intravascular Ultrasound Criteria for the Assessment of the Functional Significance of Intermediate Coronary Artery Stenoses and Comparison with Fractional Flow Reserve. Am J Cardiol 2001;87:136-141

[7] Abizaid A, Mintz GS, Pichard A, et al. Clinical, Intravascular Ultrasound, and Quantitative Angiographic Determinants of the Coronary Flow Reserve Before and After Percutaneous Transluminal Coronary Angioplasty. Am J Cardiol 1998;82:423-428

[8] Nishioka T, Amanullah A, Luo H, et al. Clinical Validation of Intravascular Ultrasound Imaging for Assessment of Coronary Stenosis Severity. Comparison With Stress Myocardial Perfusion Imaging. J Am Coll Cardiol 1999;33:1870-1878 
[9] Takagi A, Tsurumi Y, Suzuki K, et al. Clinical Potential of Intravascular Ultrasound for Physiological Asessment of Coronary Stenosis. Circulation 1999;100:250-255

[10] Lee Ch, Tai B, Soon Ch, Low A, Poh K, Yeo T, Lim G, Yip J, Omar A, Teo S, Tan H. New set of intravascular ultrasound-derived anatomic criteria for defining functionally significant stenoses in small coronary arteries (results from intravascular diagnostic evaluation of atherosclerosis in Singapore [IDEAS] Study). Am J Cardiol 2010;105:1378-1384

[11] Abizaid A, Mintz GS, Mehran R, et al. Long Term Follow-up After Percutaneous Transluminal Coronary Angioplasty Was Not Performed Based on Intravascular Ultrasound Findings. Circulation 1999;100:256-261

[12] Jasti V, Ivan E, Yalamanchili V, et al. Correlation between fractional flow reserve and intravascular ultrasound in patients with an ambiguous left main coronary artery stenosis. Circulation 2004;110:2831-2836).

[13] Abizaid A, Mintz GS, A.Abizaid, et at. One-Year Follow-up After Intravascular Ultrasound Assessment of Moderate Left Main Coronary Artery Disease in Patients With Ambiquous Angiograms. J Am Coll Cardiol 1999;34:707-715

[14] Kang SJ, Lee JY, Ahn JM, Mintz GS, Kim WJ, Park DW, Yun SCh, Lee SW, Kim YH, Lee ChW, Park SW, Park SJ. Validation of Intravascular Ultrasound-Derived Parameters With Fractional Flow Reserve for Assessment of Coronary Stenosis Severity. Circulation: Cardiovascular Interventions 2011; 4: 65-71

[15] Ben-Dor I, Torguson R, Gaglia MA, Gonzales MA, Maluenda G, Bui AB, Xue Z, Satle LF, Suddath WO, Lindsay J, Pichard AD, Waksman R. Correlation between fractional flow reserve and intravascular ultrasound lumen area in intermediate coronary artery stenosis. Eurointervention 2011;7:225-233

[16] Ahn JM. Kang SJ, Mintz GS, Oh JH, Kim WJ, Lee JY, Park DW, Lee SW, Kim YH, Lee ChW, Park SW, Moon DH, Park SJ. Validation of minimal lumen area measured by intravascular ultrasound for assessment of functionally significant coronary stenoses. JACC Cardiovasc Interv 2011;4:665-671

[17] Stone GW, Maehara A, Lansky A, de Bruyne B, Cristea E, Minth GS, Mehran R, McPherson J, Farhat N, Marso SP, Parise H, Templin B, White R, Zhankg Z,Serruys PW. A prospective natural-history study of coronary atherosclerosis.NEJM 2011;364:226-235

[18] Fearon WF, Bornschein B, Tonino PA, Gothe RM, Bruyne BD, Pijls NH, Siebert U; Fractional Flow Reserve Versus Angiography for Multivessel Evaluation (FAME) Study Investigators. Economic evaluation of fractional flow reserve-guided percutaneous coronary intervention in patients with multivessel disease.Circulation 2010;122:2545-50

[19] Nam ChW, Yoon HJ, ChoYK, Park HS, Kim H, Hur SH, Kim YN, Chung IS, Koo BK, Tahk SJ, Fearon WF, Kim KB. Outcomes of percutaneous coronary intervention in intermediate coronary artery disease. Fractional flow reserve-guided versus intravascular ultrasound-guided. JACC Cardiol Intv 2010;3:812-817

[20] Ricciardi M, Meyers S, Choi K, et al. Angiographically silent left main disease detected by intravascular ultrasound: A marker for future adverse cardiac events. Am Heart J 2003;146:507-512

[21] Hermiller JB, Buller CE, Tenaglia AN, et al. Unrecognized left main artery disease in patients undergoing interventional procedures. Am J Cardiol 1993;71:173-176 
[22] Ge J, Liu F, Görge G, et al. Angiographically „silent“ plaque in the left main coronary artery detected by intravascular ultrasound. Coronary Artery disease 1995;6:805-810

[23] Russo RJ, Wong SC, Marchant D, et al. Intravascular ultrasound-directed clinical decision making in the setting of an inconclusive left main coronary angiogram: final results from the Left Main IVUS Registry. Circulation 2004;108:IV-462

[24] Suter Y, Schoenenberger AW, Toggweiler S, Jamshidi P, Resink T, Erne P. Intravascular ultrasound-based left main coronary artery assessment: comparison between pullback from left anterior descending and circumflex arteries. J Invasive Cardiol 2009;21:457-460

[25] Oviedo C, Maehara A, Mintz GS, Tsujita K, Kubo T, Doi H, Castellanos C, Lansky AJ, Mehran R, Dangas G, Leon MB, Stone G, Templin B, Araki H, Ochiai M, Moses JW. Is accurate intravascular ultrasound evaluation of the left circumflex ostium from a left anterior descending to left main pullback possible? Am J Cardiol 2010;105:948-95

[26] Park SJ, Kim YH, Park DW, Lee SW, Kim WJ, Suh J, Yun SCh, Lee CHW, Hong MK, Lee JH, Park SW; MAIN-COMPARE Investigators. Impact of intravascular guiaxdance on Long-term mortality in stenting for unprotected left main coronary artery stenosis. Circ Cardiovasc Interv 2009;2:167-177

[27] Kotani J, Mintz GS, Rai P, et al. Intravascular ultrasound assessment of angiographic filling defects in native coronary arteries: Do they always contain thrombi ? JACC 2004;44:2087-2089

[28] Kobayashi Y, De Gregorio J, Kobayashi N, et al. Stented segments length as an independent predictor of restenosis. J Am Coll Cardiol 1999:34:651-659

[29] Grewal J, Ganz P, Selwyn A, et al. Usefulness of intravascular ultrasound in preventing stenting of hazy areas adjacent to coronary stents and its support of support spotstenting. Am J Cardiol 2001;87:1246-1249

[30] Ziada KM, Tuzcu EM, De Franco AC, et al. Intravascular ultrasound assessment of the prevalence and cause of angiographic "haziness" following high-pressure coronary stenting. Am J Cardiol 1997;80:116-121

[31] Maehara A, Mintz GS, Ahmed JM, et al. An intravascular ultrasound classification of angiographic coronary artery aneurysms. Am J Cardiol 2001;88:365-370

[32] Mizuno K, Kurita A, Imazeki N. Pathological findings after percutaneous transluminal coronary angioplasty. Br Heart J 1984;52:588-590

[33] Honey J, Mahon D, Jain A, et al. Morphological effects of coronary balloon angioplasty in vivo assessed by intravascular ultrasound imaging. Circulation 1992;85:10121025

[34] Stone G, Hodgson J, Goar F, et al. Improved procedural results of coronary angioplasty with intravascular ultrasound-guided balloon sizing. The CLOUT Pilot Trial. Circulation 1997;95:2044-2052

[35] Haase K, Athanasiadis A, Mahrholdt H, et al. Acute and one year follow-up results after vessel size adapted PTCA using intravascular ultrasound. Eur Heart J 1998;19:263-272

[36] Schroeder S, Baumbach A, Haase K, et al. Reduction of restenosis by vessel size adapted percutaneous transluminal coronary angioplasty using intravascular ultrasound. Am J Cardiol 1999;83:875-879 
[37] Abizaid A, Pichard A, Mintz GS, et al. Acute and long-term results of an intravascular ultrasound-guided percutaneous transluminal coronary angioplasty/provisional stent implantation strategy. Am J Cardiol 1999;84:1298-1303

[38] Schiele F, Meneveau N, Gilard M, et al. Intravascular ultrasound-guided balloon angioplasty compared with stent. Immediate and 6-month results of the multicenter, randomized balloon equivalent to stent study (BEST). Circulation 2003;107:545-551

[39] Frey A, Hodgson J, Muller Ch, et al. Ultrasound-guided strategy for provisional stenting with focal balloon combination catheter. Results from the randomized strategy for intracoronary ultrasound-guided PTCA and stenting (SIPS) trial. Circulation 2000;102:2497-2502

[40] Gaster A, Skjoldborg S, Larsen J, et al. Continued improvement of clinical outcome and cost effectiveness following intravascular ultrasound guided PCI: insight from a prospective, randomized study. Heart 2003;89:1043-1049

[41] Mueller Ch, Hodgson J, Schindler Ch, et al. Cost-effectiveness of intracoronary ultrasound for percutaneous coronary interventions. Am J Cardiol 2003;91:143-147

[42] Colombo A, De Gregorio J, Moussa I, et al. Intravascular ultrasound-guided percutaneous transluminal coronary angioplasty with provisional spot stenting for treatment of long coronary lesion. J Am Coll Cardiol 2001;38:1427-1433

[43] von Birgelen, Mintz GS, Eggebrecht H, et al. Preintervention arterial remodeling affects vessel stretch and plaque extrusion during coronary stent deployment as demonstrated by three-dimensional intravascular ultrasound. Am J Cardiol 2003;92:130-135

[44] Ahmed JM, Mintz GS, Weissman NJ, et al. Mechanism of lumen enlargement during intracoronary stent implantation. An intravascular ultrasound study. Circulation 2000;102:7-10

[45] Costa JR, Mintz GS, Carlier SG,Costa RA, Fujii K,Sano K, Kimura M, Lui J, Weisz G, Moussa I, Dangas G, Mehran R, Lansky AJ, Kreps EM, Collins M, Stone GW, Moses JW, MD, Leon MB. Intravascular Ultrasonic Assessment of Stent Diameters Derived from Manufacturer's Compliance Charts. Am J Cardiol 2005;96:74 -78

[46] Ponde CK, Aroney CN, McEniery PT, et al. Plaque prolapse between struts of the intracoronary Palmaz-Schatz stent: report of two cases with a novel treatment of this unusual problem. Cathet Cardiovasc Diagn 1997;40:353-357

[47] Colombo A, Hall P, Nakamura S, et al. Intracoronary stenting without anticoagulation accomplished with intravascular ultrasound guidance. Circulation 1995;91:16761688

[48] Gorge G, Haude M, Ge J, et al. Intravascular Ultrasound After low and high inflation pressure coronary artery stent implantation. J Am Coll Cardiol 1995;26:725-730

[49] Choi JW, Vardi GM, Meyers SH, et al. Role of intracoronary ultrasound after highpressure stent implantation. Am Heart J 2000;139:643-648

[50] de Jaegere P, Mudra H, Figulla $H$, et al. Intravascular ultrasound-guided optimized stent deployment. Immediate and 6 months clinical and angiographic results from the Multicenter Ultrasound Stenting in Coronaries Study (MUSIC Study). Eur Herat J 1998;19:1214-1223

[51] Blasini R, Neumann FJ, Schmitt C, et al. Restenosis rate after intravascular ultrasoundguided coronary stent implantation. Cathet Cardiovasc Diagn 1998;44:380-386 
[52] Schiele F, Meneveau N, Vuillemenot A, et al. Impact of intravascular ultrasound guidance in stent deployment on 6 month restenosis rate: a multicenter, randomized study comparing two strategies-with and without intravascular ultrasound guidance. J Am Coll Cardiol 1998;32:320-328

[53] Mudra H, di Mario C, de Jaegere P, et al. Randomized comparison of coronary stent implantation under ultrasound or angiographic guidance to reduce stent restenosis (OPTICUS study). Circulation 2001;104:1343-1349

[54] Fitzgerald PJ, Oshima A, Hayase M, et al. Final Results of the Can Routine Ultrasound Influence Stent Expansion (CRUISE) Study. Circulation 2000;102:523-530

[55] Russo RJ, Silva PD, Teirstein PS, Attubato MJ, Davidson CJ, De-Franco AC, Fitzgerald PJ, Goldberg SL, Hermiller JB, Leon MB, Ling FS, Lucisano JE, Schatz RA, Wong SC, Weissman NJ, Zientek DM;AVID Investigators. A randomized controlled triual of angiography versus intravascular ultrasound-directed bare-metal coronary stnet placement (the AVID trial). Circ Cardiovasc Interv 2009;2:113-123

[56] Oemrawsingh PV, Mintz GS, Schalij M, et al. Intravascular ultrasound guidance improves angiographic and clinical outcome of stent implantation for long coronary artery stenoses. Final results of a Randomized Comparison With Angiographic Guidance (TULIP Study). Circulation 2003;107:62-67

[57] Orford J, Denktas A, Williams B, et al. Routine intravascular ultrasound scanning guidance of coronary stenting is not associated with improved clinical outcomes. Am Heart J 2004;148:501-6

[58] Gil RJ, Pawlowski T, Dudek D, Horszczaruk G, Zmudka K, Lesiak M, Witkowski A, Ochala A, Kubica J; Investigators of Direct Stenting vs. Optimal Angioplasty trial (DIPOL). Comparison of angiographically guided direct stenting technique with direct stenting and optimal balloon angioplasty guided with intravascular ultrasound: the multicenter, randomized trial results. Am Heart J 2007;154:669-675

[59] Gaster AL, Slothuus U, Larsen J, Thayssen P, Hagfelt TH. Cost-effectiveness analysis of intravascular ultrasound guided percutaneous coronary intervention versus conventional percutaneous coronary intervention. Scand Cardiovasc J 2001;35:80-85.

[60] Gaster AL, Slothuus Skjoldborg U, Larsen J, Korsholm L, von Birgelen C, Jensen S, Thayssen P, Pedersen KE, Hagfelt TH. Continued improvement of clinical outcome and cost effectiveness following intravascular ultrasound guided PCI: insight from a prospective, randomized study. Heart 2003;89:1043-1049

[61] Katritsis D, Ioannidis J Korovessis S, et al. Comparison of myocardial fractional flow reserve and intravascular ultrasound for the assessment of slotted-tube stents. Cathet Cardiovasc Intervent 2001;52:322-326

[62] Fearon W, Luna J, Samady H, et al. Fractional flow reserve compared with intravascular ultrasound guidance for optimizing stent deployment. Circulation 2001;104:19171922

[63] Hanekamp CE, Koolen JJ, Pijls NH, Michels HR, Bonnier HJ. Comparison of quantitative coronary angiography, intravascular ultrasound, and coronary pressure measurement to assess optimum stent deployment. Circulation 1999;99:1015-21

[64] Moussa I, Moses J, di Mario C, et al. Does the specific intravascular ultrasound criterion used to optimize stent expansion have an impact on the probability of stent restenosis ? Am J Cardiol 1999;83:1012-1017 
[65] Hoffmann R, Mintz GS, Mehran R, et al. Intravascular ultrasound predictors of angiographic restenosis in lesions treated with Palmaz-Schatz stents. J Am Coll Cardiol 1998;31:43-49

[66] Hong MK, Park SW, Mintz GS, et al. Intravascular ultrasonic predictors of angiographic restenosis after long coronary stenting. Am J Cardiol 2000;85:441-445

[67] de Feyter PJ, Kay P, Disco C, et al. Reference chart derived from post-stent-implantation intravascular ultrasound predictors of 6-moth expected restenosis on quantitative coronary angiography. Circulation 1999;100:1777-1783

[68] von Birgelen C, Mintz GS, Bose D. et al. Impact of moderate lesion calcium on mechanism of coronary stenting as assessed with three-dimensional intravascular ultrasound in vivo. Am J Cardiol 2003;92:5-10

[69] Hong MK, Park SW, Lee CW, et al. Preintervention arterial remodeling as a predictor of intimal hyperplasia after intracoronary stenting: a serial intravascular ultrasound study. Clin Cardiol 2002;25:11-15

[70] Endo A, Hirayama H, Yoshida O, et al. Arterial remodeling influences the development of intimal hyperplasia after stent implantation. JACC 2001;37:70-75

[71] Shiran A, Weissman NJ, Leiboff B, et al. Effect of preintervention plaque burden on subsequent intimal hyperplasia in stented coronary lesions. Am J Cardiol 2000;86:1318-1321, 69/ Alfonso F, Garcia P, Pimental G, et al. Predictors and implications of residual plaque burden after coronary stenting: an intravascular ultrasound study. Am Heart J 2003;145:254-261

[72] Shiran A, Mintz GS, Waksman R et al. Early lumen loss after treatment of in-stent restenosis: an intravascular ultrasound study. Circulation 1998;98:200-203

[73] Okabe T, Mintz GS, Buch A, et al. Intravascular ultrasound parameters associated with stent thrombosis after drug-eluting stent deployment. Am J Cardiol 2007;4:615-620

[74] Cheneau E, Leborgne L, Mintz GS, et al. Predictors of subacute stent thrombosis. Results of a systematic intravascular ultrasound study. Circulation 2003;108:43-47

[75] Parise H, Maehara A, Stone GW, Leon MB, Mintz GS. Meta-analysis of randomized studies comparing intravascular ultrasound versus angiographic guidance of percutaneous coronary intervention in pre-drug-eluting stent era. Am J Cardiol 2011;107:374-382

[76] Sousa JE, Costa MA, Abizaid A, et al. Lack of neointimal proliferation after implantation of sirolimus-coated stents in human coronary arteries: a quantitative coronary angiography and three-dimensional intravascular ultrasound study. Circulation 2001;103:192-195

[77] Holmes DR Jr, Leon MB, Moses JW, Popma JJ, Analysis of 1-year clinical outcomes in the SIRIUS trial: a randomized trial of a sirolimus-eluting stent versus a standard stent in patients at high risk for coronary restenosis. Circulation 2004;109:634-40

[78] Hong MK, Minz GS, Lee ChW, et al. Paclitaxel coating reduces in-stent intimal hyperplasia in human coronary arteries. A serial volumetric intravascular ultrasound analysis from the aSian Paclitaxel-Eluting Stent Clinical Trial (ASPECT). Circulation 2003;107:517-520

[79] Moses JW, Leon MB, Popma JJ, Fitzgerald PJ, Holmes DR, O'Shaughnessy C, Caputo RP, Kereiakes DJ, Williams DO, Teirstein PS, Jaeger JL, Kuntz RE. Sirolimus-eluting stents versus standad stents in patients with stenosis in a native coronary artery. $N$ Engl J Med. 2003; 349:1315-1323 
[80] Stone GW, Ellis SG, Cox DA, Hermiller J, O'Shaughnessy C, Mann JT,Turco M, Caputo R, Bergin P, Greenberg J, Popma JJ, Russel ME. Apolymer-based, paclitaxel-eluting stent in patients with coronary artery disease. N Engl J Med. 2004;350:221-230

[81] Cervinka P, Costa MA, Angiolillo DJ, Spacek R, Bystron M, Kvasnak M, Veselka J, Nadna H, Futamatsu K. Head-to-Head Comparison Between Sirolimus-Elutingand Paclitaxel-Eluting Stents in Patients With Complex Coronary Artery Disease: An Intravascular Ultrasound Study. Catheterization and Cardiovascular Interventions 2006;67:846-851

[82] Sonoda S, Morino Y, Jako J, et al. Impact of final stent dimensions on long-term results following sirolimus-eluting stent implantation. Serial intravascular ultrasound analysis from the SIRIUS trial. J Am Coll Cardiol 2004;43:1959-1963,

[83] Moses JW, Dangas G, Mehran R, Mintz GS. Drug-eluting stents in real world: how intravascular ultrasound can improve clinical outcome. Am J Cardiol 2008;102[suppl]:24J-28J

[84] Jeremias A, Sylvia B, Bridges J, et al. Stent thrombosis after successful sirolimus-eluting stent implantation. Circulation 2004;109:1930 -2

[85] Fuji K, Carlier SG, Mintz GS, et al. Stent under expansion and residual reference segment stenos are related to stent thrombosis after sirolimus- eluting stent implantation: an intravascular ultrasound study. JACC 2005;45:995-998

[86] Uren NG, Schwarzacher P, Metz JA, et al. Predictors and outcomes of stent thrombosis. An intravascular ultrasound registry. Eur Heart J 2002;23:124-132

[87] Cheneau E, Leborgne L, Mintz GS, et al. Predictors of subacute stent thrombosis. Results of a systematic intravascular ultrasound study. Circulation 2003;108:43-47

[88] Cutlip DE, Baim DS, Ho KK, Popma JJ, Lansky AJ, Cohen DJ, Carrozza JP Jr, Chauhan MS, Rodriguez O, Kuntz RE. Stent thrombosis in the modern era: a pooled analysis of multicenter coronary stent clinical trials. Circulation 2001;103: 1967-1971.

[89] Roy R, Steinberg DH, Sushinsky SJ, Okabe T, Slottow TP, Kaneshige K, Xue Z, Satler LF, Kent KM, Suddath WO, Pichard AD, Weissman NJ, Lindsay J, Waksman R. The potencial clinical utility of intravascular ultrasound guidance in patients undergoing percutaneous coronary intervention with drug-eluting stents. European Heart J 2008;29:1851-1857

[90] Claessen BE, Mehran R, Mintz GS, Weisz G, Leon MB, Dogan O, de Ribamar Costa J, Stone GW, Apostolidou I, Morales A, Chantziara V, Syros G, Sanidas E, Xu K, Tijssen JG, Henriques JP, Piek JJ, Moses JW, Meahara A, Dangas DG. Impact of intravascular ultrasound imaging on early and late clinical outcomes following percutaneous coronary intervention with drug-eluting stents. JACC Cardiovasc Interv. 2011;4:974-981

[91] Hur SH, Kang SJ, Kim YH, Ahn JM, Park DW, Lee SW, Yun SC, Lee CW, Park SW, Park SJ. Impact of intravascular ultrasound-guided percutaneous coronary intervention on long-term clinical outcomes in a real world population. Catheter Cardiovasc Interv 2011; ahead of print

[92] Degertekin M, Serruys PW, Tanabe K, et al. Long-term follow-up of incomplete stent apposition in patients who received sirolimus-eluting stent for de novo coronary lesions. An intravascular ultrasound analysis. Circulation 2003;108:2747-2750

[93] Serruys PW, Degertekin M, Tanabe K, et al. Intravascular ultrasound findings in the multicenter, randomized, double-blind RAVEL (RAndomized study with the 
sirolimus-eluting VElocity balloon-expandable stent in the treatment of patients with de novo native coronary artery Lesions) trial. Circulation. 2002;106:798-803

[94] Shah VM, Mintz GS, Apple S, et al. Background incidence of late malapposition after bare-metal stent implantation. Circulation 2002;106:1753-175

[95] Tanabe K, Serruys P, Degertekin M, et al. Incomplete stent apposition after implantation of paclitaxel-eluting stents or bare metal stents. Insights from the randomized TAXUS II trial. Circulation 2005;111:900-90

[96] Hong MK, Mintz GS, Lee ChW, Park DW, Park KM, Lee BK, Kim YH, Song JM, Han $\mathrm{KH}$, Kang DH, Cheong SS, Song JK, Kim JJ, Park SW, Park SJ. Late Stent Malapposition After Drug-Eluting Stent Implantation An Intravascular Ultrasound Analysis With Long-Term Follow-Up. Circulation 2006; 113:414-419

[97] Kimura M, Mintz GS, Carlier S, Takebayashi H, Fujii K, Sano K, Yasuda T, Costa RA, Costa JR, Quen J, et al. Outcome after acute incomplete sirolimus-eluting stent apposition as assessed by serial intravascular ultrasound. Am J Cardiol 2006;98: 436-442

[98] Cook S, Wenaweser P, Togni M, et al. Incomplete stent apposition and very late stent thrombosis after drug-eluting stent implantation. Circulation 2007;115:2426 -36

[99] Hassan AK, Bergheanu SC, Stijnen T, van der Hoeven BL, Snoep JD, Plevier JW, Schalij MJ, Wouter Jukema J.Late stent malapposition risk is higher after drug-eluting stent compared with bare-metal stent implantation and associates with late stent thrombosis. European Heart Journal 2010;31:1172-1180

[100] Alfonso F, Pérez-Vizcayno MJ, Ruiz M, Suarez S, Cazares M, Hernandez R, Escaned J, Bañuelos C, Jiménez-Quevedo P, Macaya C. Coronary Aneurysms After DrugEluting Stent Implantation Clinical, Angiographic, and Intravascular Ultrasound Findings. J Am Coll Cardiol 2009;53:2053-60

[101] Aliabadi D, Tilli FV, Bowers TR, Benzuly KH, Safian RD, Goldstein JA, et al. Incidence and angiographic predictors of side branch occlusion following high-pressure intracoronary stenting. Am J Cardiol 1997;:80:994-997

[102] Furukawa E, Hibi K, Kosuge M, et al. Intravascular Ultrasound Predictors of side branch occlusion in bifurcation lesions after percutaneous coronary intervention. Circulation Journal 2005;69:325-330

[103] Stankovic G, Darremont O, Ferenc M, Hildick-Smith D, Louvard Y, Albiero R,e t al. Percutaneous coronary intervention for bifurcation lesions: 2008 consensus document from the fourth meeting of the European Bifurcation Club. Eurointervention 2009;5:39-49

[104] Medina A, Martin P, de Lezo JS, Novoa J, Melian F, Hernandez E, de Lezo JS, Pan M, Burgos L, Amador C, Morera O, Garcia A. Ultrasound study of the prevalence of plaque at the carina in lesions that affect the coronary bifurcation: implications for treatment with provisional stent. Rev Esp Cardiol 2011;64:43-50

[105] De Lezo JS, Medina A, Martin P, et al. Ultrasound findings during percutaneous treatment of bifurcated coronary lesions. Rev Esp. Cardiol. 2008;61:930-935

[106] Kang SJ, Mintz GS, Kim WJ, Lee JY, Park DW, Lee SW, Kim YH, Lee ChW, Park SW, Park SJ. Preintervention angiographic and intravascular ultrasound predictors for side branch compromise after a single-stent crossover technique. Am J Cardiol 2011 ahead of prints 
[107] Fujii K, Kobayashi Y, Mintz GS, et al. Dominant contribution of negative remodeling to development of significant coronary bifurcation narrowing. Am J Cardiol 2003;92: 59-61

[108] Ito S, Suzuki T, Ito T, Katoh O, Ojio S, Sato H, Ehara M, Suzuki T,Kawase Y, Myoishi M, Kurokawa R, Ishihara Y, Suzuki Y, Sato K, Toyama S, Fukutomi T, Itoh M. Novel Technique Using Intravascular Ultrasound-Guided Guidewire Cross in Coronary Intervention for Uncrossable Chronic Total Occlusions. Circ J 2004; 68: $1088-1092$

[109] Nishida T, Colombo A, Briguori C, et al. Outcome of nonobstructive residual dissections detected by intravascular ultrasound following percutaneous coronary intervention. Am J Cardiol 2002;89:1257-1262

[110] Sheris SJ, Canos MP, Weissman NJ, et al. Natural history of intravascular ultrasounddetected edge dissections from coronary stent deployment. Am Heart J 2000;139:59-63

[111] Maehara A, Mintz GS, Bui AB, et al. Incidence, morphology, angiographic findings, and outcomes of intramural hematomas after percutaneous coronary interventions: an intravascular ultrasound study. Circulation 2002;105:2037-2042 


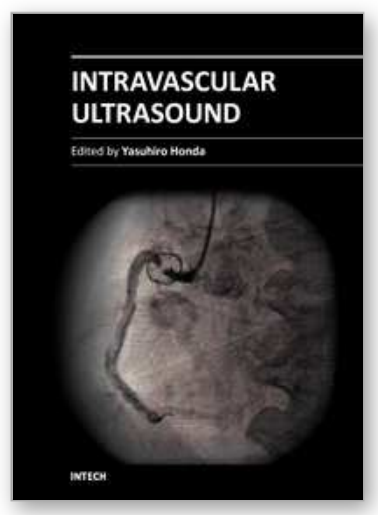

\author{
Intravascular Ultrasound \\ Edited by Dr. Yasuhiro Honda
}

ISBN 978-953-307-900-4

Hard cover, 207 pages

Publisher InTech

Published online 01, February, 2012

Published in print edition February, 2012

Intravascular ultrasound (IVUS) is a cardiovascular imaging technology using a specially designed catheter with a miniaturized ultrasound probe for the assessment of vascular anatomy with detailed visualization of arterial layers. Over the past two decades, this technology has developed into an indispensable tool for research and clinical practice in cardiovascular medicine, offering the opportunity to gather diagnostic information about the process of atherosclerosis in vivo, and to directly observe the effects of various interventions on the plaque and arterial wall. This book aims to give a comprehensive overview of this rapidly evolving technique from basic principles and instrumentation to research and clinical applications with future perspectives.

\title{
How to reference
}

In order to correctly reference this scholarly work, feel free to copy and paste the following:

T. Kovarnik and J. Horak (2012). IVUS Guided PCI, Intravascular Ultrasound, Dr. Yasuhiro Honda (Ed.), ISBN: 978-953-307-900-4, InTech, Available from: http://www.intechopen.com/books/intravascular-ultrasound/ivusguided-pci

\section{INTECH}

open science | open minds

\section{InTech Europe}

University Campus STeP Ri Slavka Krautzeka 83/A 51000 Rijeka, Croatia Phone: +385 (51) 770447 Fax: +385 (51) 686166 www.intechopen.com

\section{InTech China}

Unit 405, Office Block, Hotel Equatorial Shanghai No.65, Yan An Road (West), Shanghai, 200040, China 中国上海市延安西路65号上海国际贵都大饭店办公楼 405 单元 Phone: +86-21-62489820

Fax: $+86-21-62489821$ 
(C) 2012 The Author(s). Licensee IntechOpen. This is an open access article distributed under the terms of the Creative Commons Attribution 3.0 License, which permits unrestricted use, distribution, and reproduction in any medium, provided the original work is properly cited. 